\title{
Modeling of defects, dopant diffusion and clustering in silicon
}

\author{
Maria Aboy - I. Santos • L. Pelaz • L.A. Marqués - P. López
}

Received: date / Accepted: date

\begin{abstract}
Ion implantation is a very well established technique to introduce dopants in semiconductors. This technique has been traditionally used for junction formation in integrated circuit processing, and recently also in solar cells fabrication. In any case, ion implantation causes damage in the silicon lattice that has adverse effects on the performance of devices and the efficiency of solar cells. Alternatively, damage may also have beneficial applications as some studies suggest that small defects may be optically active. Therefore it is important an accurate characterization of defect structures formed upon irradiation. Furthermore, the technological evolution of electronic devices towards the nanometer scale has driven the need for the formation of ultra-shallow and low-resistive junctions. Ion implantation and thermal anneal models are required to predict dopants placement and electrical activation. In this article, we review the main models involved in process simulation, including ion implantation, evolution of point and extended defects and dopant-defect interactions. We identify different regimes at which each type of defect is more relevant and its inclusion in the models becomes crucial. We illustrate in some examples the use of atomistic modeling techniques to gain insight into the physics involved in the processes as well as the relevance of the accuracy of models.
\end{abstract}

Keywords Defects · Dopants · Silicon · Modeling

\section{Introduction}

The most common technique used to selectively introduce dopants in the Si substrate and define junctions is

Departamento de Electrónica, E.T.S.I. de Telecomunicación, Universidad de Valladolid, 47011 Valladolid, Spain ion implantation because it allows a precise control of the amount and distribution of dopants [1]. This technique has been traditionally used for junction formation in logic and memory devices, and recently is taking a renewed interest also in solar cells fabrication [2]. As the energetic incoming ions penetrate into the substrate the Si crystal lattice is damaged. Moreover, generally the as-implanted dopant atoms do not lie in substitutional lattice sites and they are electrically inactive. Subsequent thermal anneals are required to heal the crystal damage and to place the dopants in substitutional sites. The maximization of drive current for higher switching speed in logic and memory devices demands high dopant activation levels [3]. Additionally, lattice damage usually introduces energy levels into the semiconductor band-gap and this usually has detrimental influence in the device performance. In logic and memory devices, residual defects during silicon processing are required to be fully eliminated as they increase leakage current leading to unsustainable power consumption [4]. The minimization of defects acting as recombination centers for photogenerated electron-hole pairs is essential to achieve good conversion efficiency in solar cells [5]. However, damage may also have beneficial applications as some studies suggest that small defect clusters may be optically active bestowing enhanced optical performance on silicon $[6,7]$. Therefore, it is of critical importance not only to know the final dopant profiles, but also to quantify the amount and morphology of the damage formed upon irradiation since it will influence the final performance of devices.

Modeling has become an essential step for the understanding of physical mechanisms involved in junction formation and for process evaluation and optimization. Important and challenging issues in the area of the front-end process modeling are the diffusion and inter- 
actions of dopants and defects [3]. These processes are highly transient and its dynamics needs to be captured by models in order to define the optimum processes that provide maximal dopant activation with minimal diffusion for ultra-shallow junctions required for nanodevices. Predictive process simulation has stimulated the development of detailed models about dopant diffusion, evolution of extended defects, and formation and dissolution of dopant-defect clusters.

Most process simulators used in industrial applications are based on continuum methods, as it is the case of FLOOPS [8]. In this kind of simulators the physics of the system is formulated as a series of partial differential equations for each particle type considered to be relevant in the process $[9,10]$. Continuum simulators are fast and allow the consideration of big systems by adjusting the grid used for the spatial discretization. However, this advantage is reduced as the device size shrinks to nanometric scale where the atomistic nature of the material arises and complex physical interactions show up. The use of a very refined grid and the addition of more equations to include such new effects is computationally expensive, which slows down the resolution of the problem using continuum methods. Then atomistic simulation techniques become a good alternative even for industrial applications [11-14]. The dynamics of the system can be also simulated from an atomistic point of view by the use of Kinetic Monte Carlo (KMC) techniques. This method allows the simulation of device structures at a macroscopic scale, providing an atomic description of the material and allowing a fast development of new models. KMC simulates the kinetics of defects and dopants by modeling their diffusion and interactions $[15,16]$. In non-lattice KMC models, atoms in the perfect lattice are not simulated, and consequently system sizes of hundreds of nanometers can be treated using average computers, as it is the case of the code DADOS used for front-end process modeling [16].

In order to simulate the dynamics of a system with KMC or continuum methods is necessary to provide the values of the activation energies and prefactors for each one of the reactions that may take place (defect formation and dissolution, dopant-defect interactions... ). Only in a few cases these parameters can be obtained directly from experiments, due to the difficulty of extracting information at the atomic scale. Generally, this kind of information can be obtained from more fundamental atomistic simulation methods, where the system under study is treated as a set of interacting particles. There exists a number of different techniques that can be used for these purpose. Some of them describe the interactions among the particles on the basis of Quantum Mechanics, known as ab initio simulations, such as those based on the Density Functional Theory (DFT) $[17,18]$, or with some simplifications such as Tight Binding (TB) simulations [19-21]. Other techniques omit the electronic description and account for the effective influence of the electrons over the atoms through analytical expressions, called empirical potential. Once that the interactions between the particles are defined, static calculations (e.g. structure relaxations) or Molecular Dynamics (MD) simulations can be performed in order to extract the required information from the system under study (formation energies, energy barriers... ). Each one of these simulation techniques is suitable for providing information of different aspects of the system under study, from electronic related features (DFT, TB) to the evolution of atomic structures during annealing (empirical potentials), and at different space and time scales. Si process modeling requires a multi-scale simulation scheme where several techniques must be used together for a complete description of the relevant phenomena involved in front-end processes.

In this article we review some of the most relevant models involved in the prediction of defect distribution and $\mathrm{B}$ diffusion and electrical activation in Si. We focus our study in the case of B since it is one of the most common dopants used for the formation of $p$-type regions and it presents a number of intriguing effects associated with nonequilibrium B-defect interactions that are responsible for resulting active dopant profiles. This work is organized as follows. Section 2 is specifically devoted to the modeling of defects generated by ion implantation. In Section 3 we center our attention on the analysis and modeling of B-defect interactions. Both sections are complemented with some practical applications in order to clarify main aspects of models. Finally, in Section 4, main conclusions of this work are reported.

\section{Ion implantation and defects}

Ion implantation is the technique preferred nowadays to introduce dopants for fabricating junctions of devices since it is a well established technique and it provides a precise control of the distribution and concentration of the dopants in the Si substrate [1]. In this technique dopant atoms are first ionized, then accelerated through an electric field, and finally the resulting beam of ions is oriented toward the region to be doped. When the energetic ions penetrate into the substrate, they start to collide with its atoms until they come to rest. These collisions can produce permanent displacements of the substrate atoms from their perfect lattice positions. If the energy transferred to target atoms is high enough, they can initiate a subcascade leaving behind a vacancy and generating a Si interstitial where they stop, in addition 
to possible displacements during the subcascade. During annealing treatments, Si interstitial-vacancy pairs generated in each implantation cascade (called Frenkel pairs) quickly recombine to restore the damaged lattice (during the implant itself and the initial stages of anneals), leaving approximately one Si intersitial per implanted ion which cannot be annealed out immediately ("+1" model) [22]. These excess interstitials that have no vacancies to recombine with, condense into $\mathrm{Si}$ interstitial clusters, $\{113\}$ defects and eventually dislocation loops $[23,24]$. These defects survive for a long time until they are annihilated at the Si surface, sustaining a local supersaturation of Si interstitials (interstitial concentration compared to that in equilibrium) by emitting and recapturing interstitials during continued annealing (Ostwald ripening) [25]. Additionally, the interactions of dopants with the excess of Si interstitials and vacancies (generated during the implantation, or released by extended defects) result in mobile dopant species and dopant-defect agglomerates. This has severe adverse consequences on the Si based devices as dopant diffusivity is enhanced and dopant activation is reduced compared to equilibrium values [26-29, 15,25 , 30-32]. These effects are transient because defect supersaturation evolves toward equilibrium.

The lattice can be completely amorphized if the implant dose is high enough [33]. The increasingly demand for highly doped and ultra-shallow junctions has extended the use of low-temperature solid phase epitaxial regrowth (SPER) of preamorphized Si since it has been proved to result in metastable high activation levels of dopants with minimal dopant diffusion $[34,35]$. This approach also benefits from the complete suppression of channeling of light dopant beams, since it is implanted in amorphous $\mathrm{Si}$ (a-Si). In the case of amorphizing implants, a very large amount of damage accumulates after the high dose rate implant leading to the formation of an amorphous layer (a-layer). During its subsequent regrowth (tipically during low-temperature annealing), excess atoms contained in the a-layer are swept toward the surface as the interface advances and are eliminated there $[36,37]$. A band of extended defects is formed only beyond the amorphous/crystalline (a/c) interface (the so-called end-of-range (EOR) defects), thus the dose of Si interstitials stored in defects becomes lower than the implanted dose $[38,23,24]$. However, EOR defects can also have several adverse effects, for example, the dissolution of EOR defects upon subsequent anneals degrades dopant activation and junction depth [34,35,39]. Additionally, if dislocation loops are located in the depletion region of the device, a large leakage current may be induced [40].

\subsection{Point defects}

Native point defects in Si have been an important field of both theoretical and experimental research for several decades. The interest in its study continues today due to their role in a large variety of phenomena, especially in those related to the fabrication of integrated circuits (ICs). Native Si defects affect the microstructure evolution of the material during several of the manufacturing steps, and thus can alter the final performance of the device [41]. The most fundamental building blocks for microdefect formation in crystalline $\mathrm{Si}$ (c-Si) are the self-interstitial and the vacancy. These two species are the mediators for impurity diffusion and clustering $[41,42]$.

The vacancy is the simplest intrinsic point defect in $\mathrm{Si}$ : its basic form is just a missing Si atom in an otherwise tetrahedrally coordinated lattice [43]. The formation energy of the vacancy has been estimated using both theory and experiment. There is considerable uncertainty in the actual value, with various experimental estimates lying in the range of 2 to $4 \mathrm{eV}$ [44-46]. Using $a b$ initio techniques, calculated formation energies for the vacancy range from 3 to $6 \mathrm{eV}$ (see Ref. [43] and references therein). The theoretical difficulties arising for the vacancy are related, at least in part, to the subtle reconstruction of the dangling bonds, where some controversy still remains $[47,48]$. The diffusivity of vacancies has been characterized experimentally by various methods: at low temperatures directly by Electron Paramagnetic Resonance (EPR), and at high temperatures indirectly via their effect on the diffusion of dopants and metals. While vacancy migration energies determined experimentally range from 0.3 to $4 \mathrm{eV}, a b$ initio calculations predict values in the lower end, between 0.3 and $0.4 \mathrm{eV}$ [43].

The Si self-interstitial, i.e. one extra $\mathrm{Si}$ atom in the crystal lattice, is the natural counterpart of the vacancy. The study of the Si self-interstitial properties is of particular importance in Si processing. Self-interstitials have been implicated as the origin of rodlike defects observed in Czochralsky single-crystal growth, which can ultimately produce the degradation of the manufactured Si devices [49]. On the other hand, during the implantation step a large concentration of excess interstitials is introduced in the lattice. They interact with interstitial diffuser dopants, such us B, causing the so-called Transient-Enhanced Diffusion (TED) - which alters the junction depth $[50,27]$ - and dopant-defect clusters formation - which results in electrical deactivation [26, 29].

Due to its importance in Si processing, a great number of theoretical studies have been devoted to determine the configuration and energetics of the Si self- 
interstitial, as well as its diffusive behavior. These include ab initio [51-59], TB [60-66], and empirical potential calculations [67-78]. However, even when using the same calculation techniques, different authors come to different conclusions regarding the Si self-interstitial properties. The discrepancies are mainly related to the determination of the lowest formation energy configuration and to the microscopic description of the interstitialmediated diffusion mechanism. At least four different interstitial configurations have been identified: tetrahedral $(\mathrm{T})$, dumbbell $(\mathrm{D})$, hexagonal $(\mathrm{H})$ and extended (E), and calculated formation energies for the Si selfinterstitial range from 2.2 to $5.6 \mathrm{eV}$ (see Ref. [78] and references therein). Furthermore, very different mechanisms for interstitial-mediated diffusion have been proposed, with migration energies ranging from 0.1 to 1.9 eV (see Ref. [43] and references therein). In spite of such a diversity of results, Marqués et al. demonstrated (using MD with empirical potentials) that all self-interstitial configurations coexist in Si but with different concentrations, and diffusion occurs through transitions among them [78]. The macroscopic behavior for self-interstitial diffusion can be modeled by a simple description based on a unique interstitial species with an effective formation energy of $3.8 \mathrm{eV}$ and a migration barrier of $0.8 \mathrm{eV}$, in very good agreement with experiments $[46,25]$. The exact numbers do not correspond to any of the particular interstitial configurations or diffusion mechanisms, but are the result of the averaged behavior of all of them.

\subsection{Small clusters and extended defects}

Point defects interact among them giving rise to aggregates or clusters [79-81]. Generally, the formation energy of such clusters present marked oscillations for small cluster sizes (up to 10-15 defects per cluster) but for intermediate and large cluster sizes their formation energy decreases (or alternatively, their binding energy increases) with cluster size. As a consequence, their population in the Si lattice is controlled by an Ostwald ripening process: larger clusters grow at the expense of point defects freed from smaller and less stable agglomerates $[25,82]$. The study of the properties of small clusters is difficult because they are too small to be visible in Transmission Electron Microscopy (TEM) images. Moreover, they show a great variety in their atomic configurations which complicates their analysis using simulation techniques. At sizes of several hundred point defects these aggregates start becoming visible in TEM; they usually show regular atomic structures, and are generally known as extended defects [24].

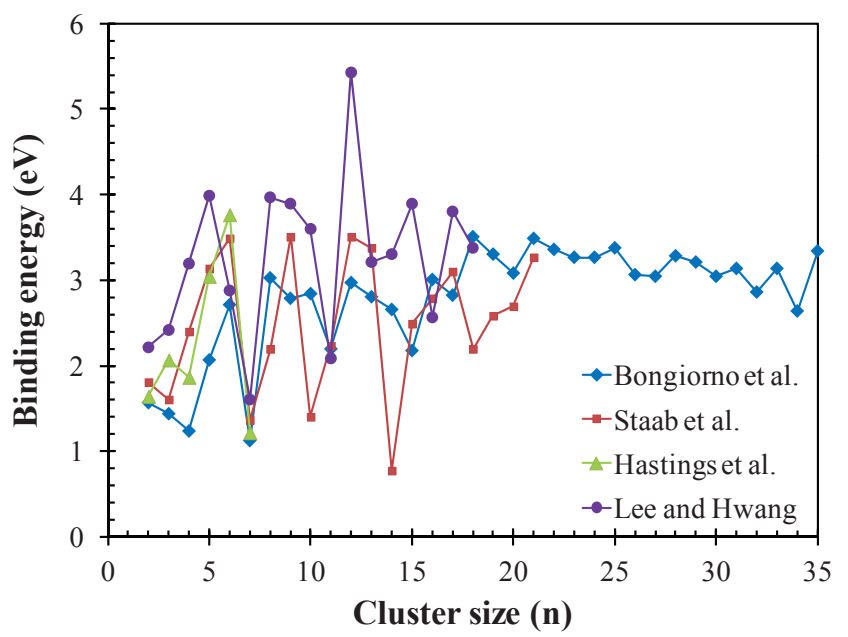

Fig. 1 Binding energy for vacancy clusters as a function of cluster size (from Refs. [83-86]).

Vacancy aggregation in Si has been studied extensively because large vacancy clusters (voids) are known to be harmful to microelectronic device yield and reliability, particularly gate-oxide integrity $[87,88]$. However, the introduction of voids in the Si lattice has been proposed as a way to reduce the interstitial supersaturation [89-92]. This controlled injection of voids, part of a more generic concept of defect engineering, allows the reduction of the anomalous diffusion of dopants such as B. Positron annihilation experiments have been used to determine the lifetime of vacancy clusters, being around $400 \mathrm{ps}$ for sizes between $\mathrm{V}_{3}$ and $\mathrm{V}_{10}$ [43]. Voids are much more stable, and have been observed directly by TEM to organize into octahedral structures aligned almost exclusively along the $\{111\}$ crystallographic planes of the Si lattice [93]. This phenomenon has been explained by the low energy of the $\mathrm{Si}(111)$ surface relative to other orientations [94]. The thermodynamics and binding properties of these vacancy clusters have been studied using quantum and classical simulation techniques $[83,95,96,86]$. In particular, for small vacancy clusters it has been found that certain sizes show greater stability, as it is the case of the $\mathrm{V}_{6}, \mathrm{~V}_{8}$ and $\mathrm{V}_{12}$ clusters (see Fig. 1), due to particular bond reconstructions in the Si lattice [83-86]. For larger sizes, binding energies tend to a value of around $3 \mathrm{eV}$, in agreement with $\mathrm{Sb}$ diffusion and Au labeling experiments [97,98].

Due to their implications in Si technology, self-interstitial aggregation in Si has attracted much attention in the literature. Ion implantation produces considerable damage in the Si lattice due to the energetic collisions of ions with the host atoms. Frenkel pairs generated in each implantation cascade typically recombine quickly and only Si interstitials generated by the implanted ions 


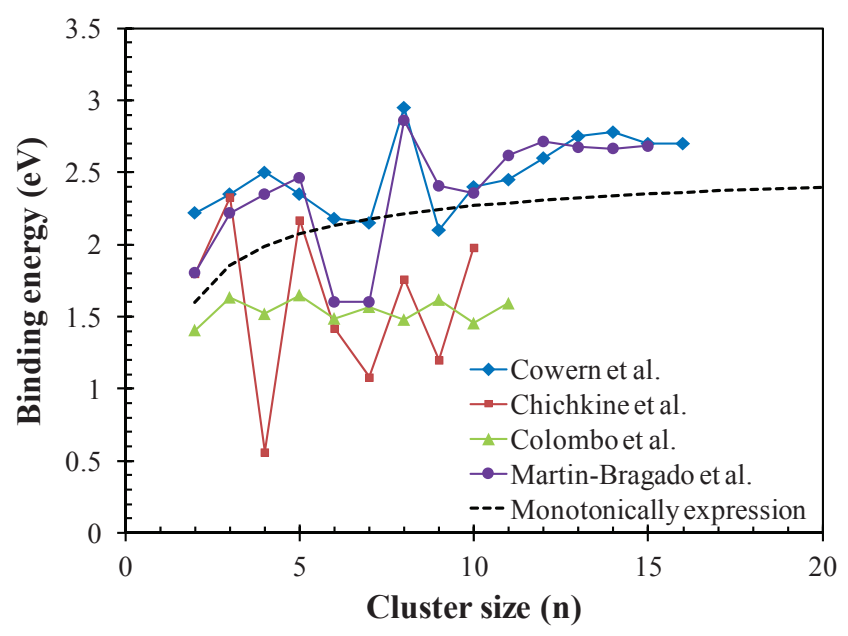

Fig. 2 Binding energy for interstitial clusters as a function of cluster size (from Refs. [25,99-101]).

survive. Residual Si interstitials agglomerate into defect clusters and extended defects. These defects act as a reservoir of Si self-interstitials that are slowly released during subsequent thermal treatments causing the TED of interstitial diffuser dopants such as B [27] and dopant clustering [26,29] which, in turn, modify the dopant profiles and device characteristics. Defects also cause carrier mobility degradation and increase leakage currents. Conversely, implantation damage in more recent years has allowed for the possibility of new $\mathrm{Si}$ based optoelectronic devices. The indirect bandgap of silicon has generally limited the attractiveness of this material for optical devices. Converting Si into a lightemitter semiconductor will make optoelectronics take advantage of the microelectronic industry technology (developed around $\mathrm{Si}$ ), and will result in a large reduction of the fabrication costs of optoelectronic devices. One of the areas which are currently being explored for efficient light emission in Si include photoluminescence (PL) through optically active defect clusters and extended defects. The demonstration of enhanced bandedge luminescence through dislocation engineering [102] or the fabrication of a sub-bandgap light emitting diode based on the introduction of small defects that enhance the radiative recombination rate of Si has attract much attention on the possibility of enabling $\mathrm{Si}$ as a light emitter [7].

From B diffusion experiments, Cowern et al. deduced the formation energy of small interstitial clusters using the concept of Ostwald ripening and the fact that the Si interstitial supersaturation, and therefore B diffusion, is related to their stability [25]. Results for the binding energy of such clusters are shown in Fig. 2, along with calculations carried out by other authors using ab initio [100], TB [99] and fitting to experiments

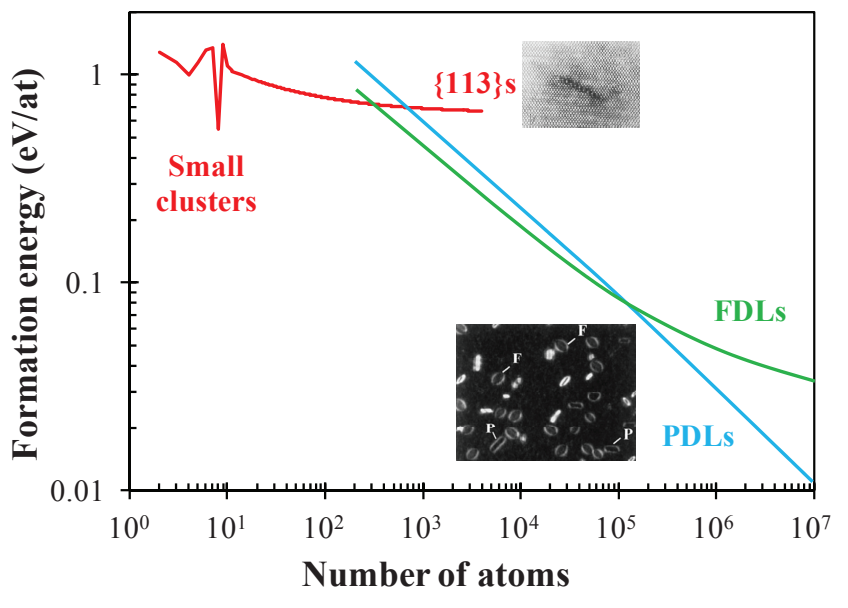

Fig. 3 Formation energy of the different types of interstitial agglomerates as a function of size (data taken from Ref. [105]; TEM images examples for the different types of interstitial defects taken from Refs. [27, 108]).

[101]. The most important finding is that oscillations of the binding energies have marked "magic" numbers for specific small cluster sizes (as with vacancy clusters). These more stable sizes correspond to configurations where atoms remain four-fold coordinated [103]. For larger sizes, of around one hundred atoms, $\{113\}$ defects start to form. Their atomic structure was determined by Takeda using TEM [104]. \{113\} defects consist of large interstitial chains along the $\langle 110\rangle$ direction, packed together along the $\{113\}$ plane, which gives this defect its name. It has been experimentally shown that $\{113\}$ defects grow in length along the $\langle 110\rangle$ direction [24]. In the process, their formation energy decreases from 0.8 to $0.65 \mathrm{eV}$ [105].

Under certain conditions, particularly for high-dose implants, $\{113\}$ defects can transform into into dislocation loops, perfect (PDLs) and faulted (FDLs) [106]. This transformation has been proposed to be due to some unfaulting reactions, as it has been shown recently by using $a b$ initio simulation techniques [107]. The formation energy of FDLs tends to $0.027 \mathrm{eV}$ with increasing size, while it tends to 0 for PDLs [105]. In Fig. 3 we show the formation energy of interstitial agglomerates as a function of size. This energy landscape determines the microstructural evolution of the material. For example, at a size of 1000 interstitials, a \{113\} would act as a sink for self-interstitials released by PDLs and as a source of self-interstitials for FDLs.

Finally, Fig. 4 gives a summary in the form of a "phase diagram" for Si interstitial defects behavior, based on the experimental observations for Si implants into Si in the energy range from 20 to $150 \mathrm{keV}$. For doses below the amorphization threshold (around $2 \times 10^{14} \mathrm{~cm}^{-2}$ ), the defect processes are only weakly dependent on the 


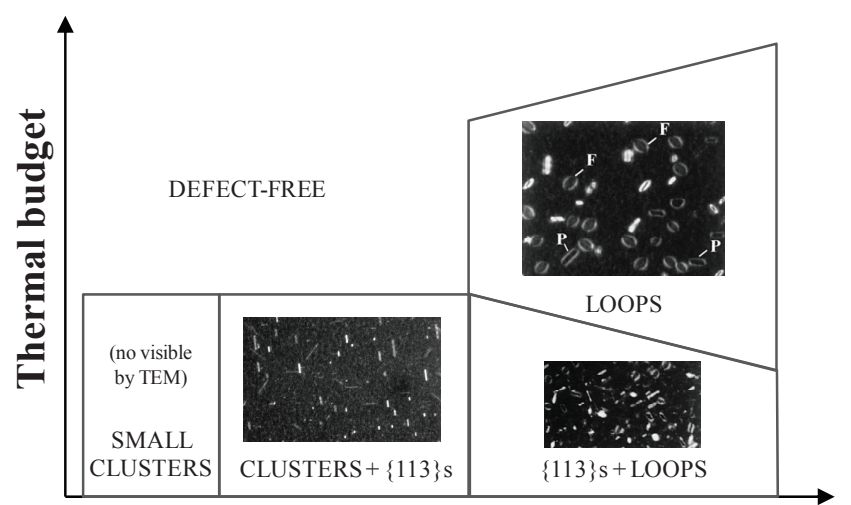

\section{Dose}

Fig. 4 Schematic diagram of the behavior of Si interstitial defects as a function of implant dose and thermal budget (TEM images examples of such defects taken from Refs. [23, 108]).

initial damage level, so that the diagram can be mapped in terms of implantation dose versus thermal annealing "budget". At implantation doses below $5 \times 10^{12} \mathrm{~cm}^{-2}$, no $\{113\}$ defects are observed. However, other experimental observations such as TED of dopants evidence the formation of Si interstitial defects at these conditions. Thus, at low implantation doses only small Si interstitial clusters form from the implantation damage, which are too small to be detected by TEM. For doses in between $5 \times 10^{12} \mathrm{~cm}^{-2}$ and $1-2 \times 10^{14} \mathrm{~cm}^{-2}$, Si interstitial clusters and $\{113\}$ defects are visible defects and they completely dissolve for a sufficiently high thermal budget (i.e., annealing time and temperature). The dissolution rate at a given temperature is dependent on implantation dose and energy $[109,110]$. Above a threshold dose of $1-2 \times 10^{14} \mathrm{~cm}^{-2},\{113\}$ defects undergo unfaulting, leading to both Frank loops and perfect dislocations. Since these dislocations are more stable than $\{113\}$ defects, significantly stronger annealing steps are needed to fully dissolve the dislocation damage.

\subsection{Applications of defects modeling to physical understanding and technlogy}

In this subsection we present some examples of physically based KMC simulations which analyze Si interstitial defects evolution under different experimental conditions as well as the influence that the accuracy of defect models has on simulation results. The formation and migration energies of the free Si self-interstitial and vacancy are taken from Ref. [46], which has been tested with KMC simulations under very different experimental conditions [15,111-114,39,115]. As it has been discussed in Subsection 2.2, there are discrepancies in the literature in the binding energies obtained for small clusters by different authors (see Figs. 1-2). These discrepancies are not very significant in the case of small vacancy clusters whereas the differences between binding energies of small Si interstitial clusters given by different authors are very significant. For instance, Chichkine et al. proposed that $I_{3}$ is rather stable compared to $I_{2}$ and $I_{4}$, whereas Cowern et al. and Colombo et al. obtained similar stability for these three configurations. In order to test the influence that these significant discrepances could have on the predictions from KMC simulations, we have compared in the following examples the simulation results obtained with different set of parameters for small Si interstitial clusters (up to 10 interstitials). In particular, we consider the oscillating binding energies for the different cluster sizes reported by Chichkine et al. [100] and by Cowern et al. [25] since these authors proposed the less and more stable small Si interstitial clusters, respectively. For larger interstitial clusters and $\{113\}$ defects we use the experimentally deduced binding energy reported by Cowern et al. [25]. For vacancy clusters we consider the oscillating binding energies calculated by Bongiorno et al. [83]. Dislocation loops do not form in the experiments under study.

\subsubsection{Si interstitial cluster related luminiscence centers.}

The optical response of luminescence centers in Si has been extensively reported [116], and the so called Wcenter in the Si PL spectra appears to be one of the best candidates to turn Si into a light emitter [7]. Then, a good control and understanding of the PL generation mechanisms associated to this center is necessary for the fabrication of Si optoelectronic devices. The Wcenter is characterized by a zone centered zero-phonon line at $1218 \mathrm{~nm}(1.018 \mathrm{eV})[116,117]$. This W-line is observed in Si that has been ion implanted and subsequently annealed at a relatively low temperature. In fact, the luminescence from the $\mathrm{W}$-center is seen to reach a maximum after a $225-275^{\circ} \mathrm{C}$ annealing and can be reduced to levels where they are no longer observed after annealing at temperatures around $450^{\circ} \mathrm{C}[6,118]$. Although different experiments have shown that the $\mathrm{W}$ line is associated to small defects generated by ion implantation $[116,117,6,118,119]$, the conditions required to optimise the luminescence from the W-center have not been clearly established. Some experiments have revealed that the $\mathrm{W}$-line production appears to be related to Si interstitial clusters with a small number of Si interstitials $[6,118,119]$, although it is not quite clear yet 
which is the specific interstitial cluster that produces them.

Taking these experimental evidences into account, different first principles studies based on the DFT approximation have investigated the properties of small $\mathrm{Si}$ self-interstitial clusters in order to elucidate the atomistic origin of the $\mathrm{W}$-center in $\mathrm{Si}$ [120-122]. Some authors have proposed the $\mathrm{W}$-center to consist of a cluster of three interstitial Si atoms $\left(I_{3}\right)[120,121]$. However, other authors have not found enough evidences to associate the $I_{3}$ cluster to the W line [122]. These discrepancies are related to the difficulties of DFT calculations for evaluating the band gap of semiconductors, which is a topic in continuous discussion (see ref. [123] and the corresponding comments and replies). Consequently, there is some uncertainty when evaluating the energy levels within the gap associated to defects, and hence on the PL lines that they will produce. Therefore, the identification at the atomic level of the W PL centers in Si still remains open. From an experimental point of view main difficulties are associated to the impossibility of experimentally characterizing the many diverse defects with different sizes and configurations that result from ion implantation and low-temperature anneals, since these typically small defects are not visible by experimental techniques (see Fig. 4).

As an alternative, KMC atomistic simulations could provide an insight into this problem since they handle dopant and defects interactions at atomic level, at the same time that their results can be directly compared to experimental data. As mentioned above, we have simulated each particular situation by using different sets of parameters for small Si interstitial clusters. Since experiments suggest that $\mathrm{W}$-line is associated to small Si interstitial clusters, the binding energies considered to perform KMC simulations could be crucial. In order to test this possibility, we performed KMC simulations for Si samples implanted with $300 \mathrm{keV}$ Si ions to a dose of $10^{12} \mathrm{~cm}^{-2}$ and later annealed at temperatures of 275,400 or $525^{\circ} \mathrm{C}$ for $2 \mathrm{~min}$, similarly to recent PL experiments reported by Charnvanichborikarn et al. [118]. In those experiments, Si samples were uniformly doped with a background $\mathrm{B}$ concentration of $9.4 \times 10^{14} \mathrm{~cm}^{-3}$ which is too low to significantly affect the evolution of implant damage (see section 3 and Ref. [124]). Experiments showed a maximum in PL intensity of $\mathrm{W}$-line at annealing temperature of around $275^{\circ} \mathrm{C}$, which decreases as the annealing temperature increases and disappears at annealing temperatures of $\sim 500^{\circ} \mathrm{C}$. This suggest that the $\mathrm{W}$-center should consist of small and quite unstable interstitial defects since it requires low thermal budget to disappear. Moreover, at these implant conditions of high energy and low dose, dam-

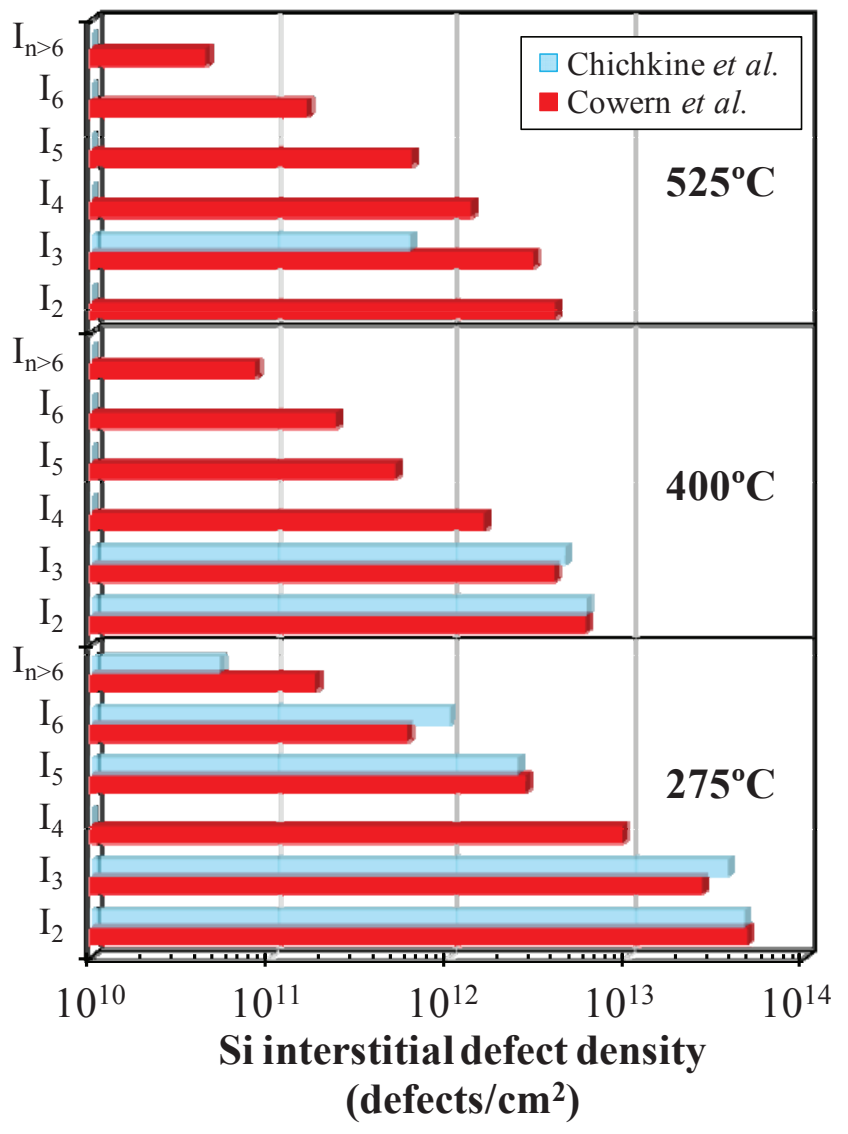

Fig. 5 Simulation results for the densities of the different Si intersititial cluster sizes for Si samples implanted with 300 $\mathrm{keV} \mathrm{Si}$ ions to a dose of $10^{12} \mathrm{~cm}^{-2}$ and subsequently annealed at 275,400 or $525^{\circ} \mathrm{C}$ for $2 \mathrm{~min}$. Simulations were performed by using binding energies for small $\mathrm{Si}$ interstitial clusters reported by Chichkine et al. [100] and Cowern et al. [25] in order to test their influence on the simulation results.

age resulting from ion implantation typically consist of small vacancy and $\mathrm{Si}$ interstitial clusters that contain a reduced number of atoms (as explained in Fig. 4), which strengthen the hypothesis of small defects as responsible for the $\mathrm{W}$-line.

Fig. 5 includes the Si interstitial cluster size distribution (density of defects for each particular Si interstitial cluster size) obtained from simulations of Si samples implanted with $300 \mathrm{keV} \mathrm{Si}$ ions to a dose of $10^{12}$ $\mathrm{cm}^{-2}$ and subsequently annealed for $2 \mathrm{~min}$ at different temperatures, similar to experiments reported by Charnvanichborikarn et al. [118]. Our simulations indicate that the two different sets of binding energies for small Si interstitial clusters lead to similar global trends. The most predominant clusters are $I_{2}$ and $I_{3}$ clusters independently on the parameter set considered. The densities of larger sizes are significantly lower, being more than one order of magnitude lower when binding energies reported by Chichkine et al. are considered. 
The densities of most of the cluster sizes decreases significantly as the annealing temperature increases, being the decrease more significant when parameters reported by Chichkine et al. are used. This reduction in the density of $\mathrm{Si}$ interstitial clusters is due to $\mathrm{Si}$ interstitialvacancy recombination as well as Si interstitial emission from interstitial clusters when it is energetically favorable (which depends on the binding energy of the Si interstitial to the particular cluster and the annealing temperature). Concerning to experiments, this reduction could be associated with the reported decrease of the luminescence from the $\mathrm{W}$-center at temperatures around $400-500^{\circ} \mathrm{C}[6,118]$. In spite of all these similarities observed between both sets of simulations, it is also evident that the results are quite sensitive to the binding energies considered for small Si interstitial clusters. The cluster size distribution is smooth if binding energies reported by Cowern et al. are considered which is in contrast to the situation observed when binding energies reported by Chichkine et al. are used. In this latter case, oscillations in binding energies are more apparent than in the values reported by Cowern et al. Thus, not all Si interstitial clusters are stable (in particular $I_{4}$ is quite unstable) which results in a rough cluster size distribution. Moreover, in this case Si interstitial clusters have practically dissolved at $525^{\circ} \mathrm{C}$ (note that, at this Si implant dose and temperature range, small Si interstitial clusters do not evolve to extended defects). Only a very small density of $I_{3}$ defects survive at this temperature, which is a very stable configuration in this parameter set. However, if parameters reported by Cowern et al. are used, considerable densities of small Si interstial clusters still remain at this temperature. This different behavior is due to the general trend proposed by Chichkine et al., with less stable small $\mathrm{Si}$ interstitial clusters compared to other sets of parameters, which favors their dissolution with relatively low thermal budgets. These considerable differences observed in simulations performed with different parameters make it difficult the identification of a particular small Si interstitial cluster configuration as responsible for the W-line. Very precise modeling for small Si interstitial clusters is necessary in order provide an accurate description of ion implantation damage that results under these particular experimental conditions.

\subsubsection{Si interstitial supersaturation and TED of interstitial diffuser dopants.}

Implant and annealing conditions typically used to fabricate junctions in Si based devices are significantly different than those required in optical applications described in the previous subsection. In particular, lower implant energies and higher implant doses are employed, which makes necessary the use of higher annealing temperatures to dissolve implant damage and restore the Si crystal lattice. These implant conditions results in larger concentrations of excess Si interstitials which favors the evolution of small interstitial clusters towards large interstitial clusters, $\{113\}$ defects and, eventually, dislocation loops (as mentioned in Fig. 4). These defects sustain a local supersaturation of Si interstitials by emitting and recapturing interstitials during continued annealing which causes TED of interstitial diffuser dopant atoms $[27,25,114]$. The transitory character of TED is associated to the dissolution of Si interstitials defects, as the interstitials are gradually lost from the damaged region through diffusion to the Si surface.

As an example, Fig. 6 plots the evolution of Si interstitial supersaturation as a function of annealing temperature and time for Si implanted with $40 \mathrm{keV} 2 \times 10^{13}$ $\mathrm{cm}^{-2} \mathrm{Si}$ ions and annealed at 600,700 and $800^{\circ} \mathrm{C}$ for times in the range $1 \mathrm{~s}$ to $20 \mathrm{~h}$. The figure includes supersaturation values reported in Ref. [25] (symbols) that were calculated from measured broadening of B profiles (due to $\mathrm{B}$ diffusion) by the method described in Ref. [25]. Similarly to previous subsection, we have analyzed the influence of different sets of binding energies for small Si interstitial clusters on the KMC simulation results (represented by lines in Fig. 6). In this case, we have also considered an expression for binding energy that monotonically rises with increasing cluster size and tends asymptotically to the experimental value of binding energy of $\{113\}$ defects $[29,25]$ (dashed line in Fig. 2). The Si interstitial supersaturation is defined as the ratio $I / I_{e q}$, where $I$ is the interstitial concentration and $I_{e q}$ is the $\mathrm{Si}$ interstitial concentration at thermal equilibrium. Experimental data show two phases of enhanced diffusion: an initial phase of ultrafast TED (high interstitial supersaturation) which is followed by a sharp drop in Si interstitial supersaturation and a lower "plateau" with near-constant supersaturation up to time $\tau$, when the supersaturation of $\mathrm{Si}$ interstitials rather abruptly decays to the equilibrium value and TED ends. The ultrafast phase persists for a much shorter time as the annealing temperature increases: at $600^{\circ} \mathrm{C}$, this phase lasts for about $1000 \mathrm{~s}$ at $700^{\circ} \mathrm{C}$, it lasts for about $10 \mathrm{~s}$, and at $800^{\circ} \mathrm{C}$ it is too short to be clearly visible in the experimental data. Simulations show that applying the constraint that binding energy of Si interstitial clusters must vary monotonically with cluster size results in supersaturation curves that vary smoothly in time, contrary to the sharp drop seen in experimental data. In contrast, the use of oscillating binding energies in simulations leads to supersaturation curves that clearly shows the two phases of 


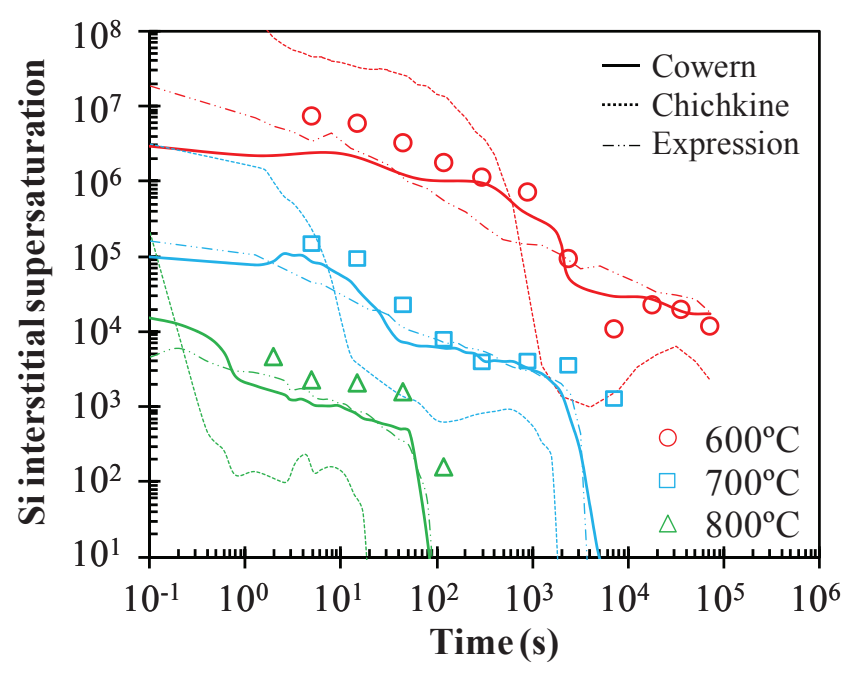

Fig. 6 Experimental (symbols) [25] and simulated (lines) time evolution of Si interstitial supersaturation for Si implanted with $40 \mathrm{keV} 2 \times 10^{13} \mathrm{~cm}^{-2} \mathrm{Si}$ ions and annealed at 600,700 and $800^{\circ} \mathrm{C}$ for times in the range $1 \mathrm{~s}$ to $20 \mathrm{~h}$. Simulations were performed by using binding energies for small $\mathrm{Si}$ interstitial clusters reported by Chichkine et al. [100], Cowern et al. [25] and the monotonically expression for binding energy included in Fig. 2 (dashed line), in order to test the influence of parameters on the simulation results.

TED (similarly to experimental data). The agreement between simulations and experimental data is better if parameters reported by Cowern et al. are considered in simulations.

According to the model proposed by Rafferty et al. [125], the free Si interstitial supersaturation, $I / I_{e q}$, in local equilibrium with defects of binding energy $E_{b}$, has an activation energy given by $\left(-E_{b}+E_{f}\right)$, being $E_{f}$ the formation energy of the Si self-interstitial from the ground state. On the other hand, the total time to dissolve the $\mathrm{Si}$ interstitial defects, and therefore to finish TED, $\tau_{T E D}$, has an activation energy given by $\left(E_{b}+E_{m}\right)$, being $E_{m}$ the migration energy of the Si selfinterstitial. This implies that unstable defects (lower $E_{b}$ ) set a high supersaturation for a short time, and vice versa, stable defects cause a lower supersaturation but subsist for longer time. Therefore, the inital phase of ultrafast TED reflects ripening of very small and unstable interstitial clusters (precursors in the nucleation of $\{113\}$ defects) whereas the lower "plateau" is associated to large interstitial clusters and $\{113\}$ defects (no dislocation loops are formed in the experiments under study). The sharp drop of Si interstital supersaturation after the initial phase is a consequence of the oscillating binding energies for small clusters which present marked peaks in binding energy for some particular cluster sizes (see Fig. 2). As discussed in Ref. [25], these particular clusters with high stability represents a sort of barrier for the growth of very small clusters to larger clusters and $\{113\}$ defects. Once the barrier is passed, a quick evolution towards larger clusters occurs which is responsible for the sharp drop observed in the Si interstitial supersaturation. The barrier effect is reduced as the annealing temperature increases because the higher flux of Si interstitials at higher temperatures enables more clusters to pass the energy maximum at shorter times. According to this reasoning, the different simulation results shown in Fig. 6 could be easily explained. The monotonically expression for binding energies does not lead to the sharp drop observed in $\mathrm{Si}$ interstitial supersaturation because there is no barrier for Si intersitial cluster growth. In the case of oscillating binding energies, the values reported by Chichkine et al. are generally lower (less stable clusters) than those proposed by Cowern et al. Thus, small clusters sustain higher Si interstitial supersaturation if parameters reported by Chichkine et al. are considered, which allows for a faster evolution towards large clusters (the sharp drop in supersaturation occurs sooner) and the total time to completely dissolve interstitial defects becomes shorter.

From Fig. 6 it is obvius that simulation results for the time evolution of supersaturation are sensitive to parameters. However, from a technological point of view, the instantaneous value of the interstitial supersaturation is not so relevant but it is much more important to predict the total dopant diffusion due to TED, which ultimately increases junction depth. From simulations it is possible to evaluate TED in terms of the time integrated Si interstitial supersaturation (as discussed in Ref. [114]), which is proportional to the diffusivity enhancement of interstitialy-diffuser dopant atoms. Fig. 7 shows the total time integrated Si interstitial supersaturation calculated from the simulation data included in Fig. 6 as a function of annealing temperature. As it is shown in Fig. 7 the total time integrated Si interstitial supersaturation (proportional to the total amount of TED) is not so sensitive to the particular model for small interstitial clusters as the Si interstitital supersaturation is. In particular, at 700 and $800^{\circ} \mathrm{C}$ the results are practically identical when oscillating binding energies reported by Cowern et al. or the monotonically expression are considered. Moreover, if parameters repoted by Chichkine et al. are considered (which led to significantly different evolution for Si interstitial supersaturation in Fig. 6), only a very slight reduction is the total amount of TED is observed at these annealing temperatures. A slightly larger difference among simulations with different parameters is observed at $600^{\circ} \mathrm{C}$, but note that at this low annealing temperature the concentration of Si interstitials is still above the equi- 


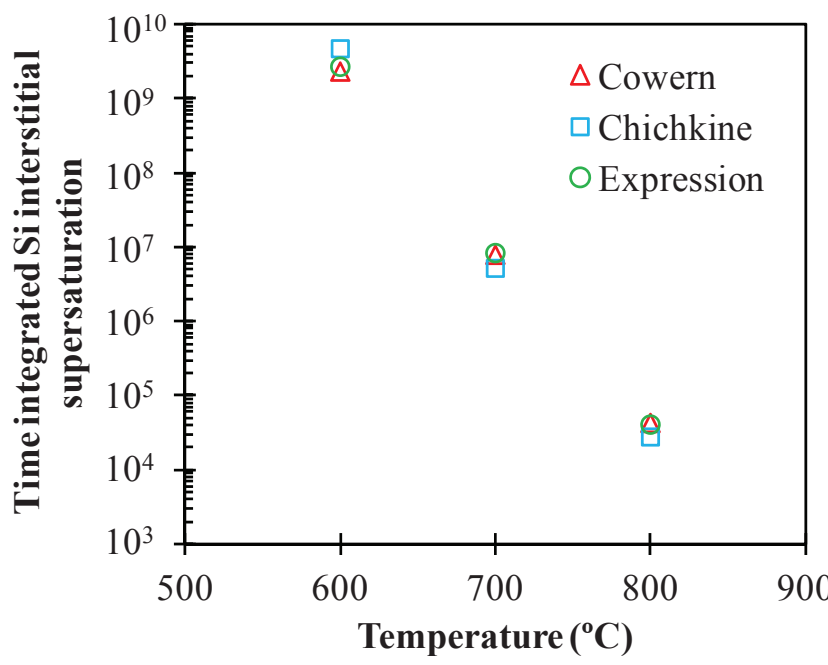

Fig. 7 Time integrated Si interstitial supersaturation calculated from simulated data included in Fig. 6. In spite of the different binding energy values considered for simulations, the total time integrated $\mathrm{Si}$ interstitial supersaturation (which is proportional to TED) is not very sensitive to parameters.

librium value after $10^{5} \mathrm{~s}$ anneal (see Fig. 6), thus TED has not finished yet. These results are in agreement with the model proposed by Rafferty et al. mentioned above [125]. According to that model, the TED to completion is given approximately by $\left(I / I_{e q}\right) \cdot \tau_{T E D}$, which corresponds to an activation energy given by $\left(E_{f}+E_{m}\right)$, thus being the total amount of TED independent on the binding energy of the Si interstitial defects. Based of that model, these authors found that the TED to completion only depends on the implant dose and the projected range of the implanted ions.

\section{Dopant diffusion and clustering}

Impurities or dopant atoms are selectively introduced into the Si substrate during some of the process steps in the fabrication of Si-based elctronic devices to modify the electrical features of Si. The most commonly used species to dope Si are the group-III acceptor impurities $\mathrm{B}, \mathrm{Ga}, \mathrm{In}$, and $\mathrm{Al}$ and the group- $\mathrm{V}$ donor impurities $\mathrm{P}$, As, and $\mathrm{Sb}$ of the Periodic Table of the Elements. These external impurities, different from atoms of the host lattice, are introduced into the Si lattice in order to improve its electrical conductivity, varying the number of free carriers in the material. Indeed, donors and acceptors tend to dissolve substitutionally in the Si lattice and become ions. If they get ionized, each donor delivers an electron to the conduction band, and each acceptor will capture an electron from the valence band leaving a defect electron (hole) behind. In this way excess electrons and holes will be delivered by ionized donors and acceptors, turning the semiconductor to $n$ type or $p$-type semiconductor, respectively. Both types of charge carriers determine the electrical characteristics of devices. For this reason, a great attention has been focused on understanding dopant behavior in $\mathrm{Si}$ and the prediction of the spatial distribution of donors and acceptors after processing is one of the main goals of process simulation.

Two relevant material properties are important for Si processing: solid solubility of dopants and dopant diffusivity. The first one determines the maximum concentration which can be incorporated into the substitutional sites of the host lattice under equilibrium thermodynamic conditions without inducing a phase transition. Typically dopants need to dissolve substitutionally to be electrically active. Therefore, to achieve high carrier concentrations in $\mathrm{Si}$ high solid solubility values for dopants are required. In addition, dopant diffusivity is desirable to be low to avoid the broadening of dopant profiles during thermal processes. As is the most common $n$-type dopant used for the formation of $n$-type regions in $\mathrm{Si}$, due to its low diffusivity and its high solid solubility [43]. In the case of $p$-type dopants, $\mathrm{B}$ is the preferred dopant to form $p$ type regions in Si due to its very high solid solubility compared to other $p$-type dopants, although it is a quite fast diffuser (which complicates the control of junction depth) [43]. These two equilibrium properties ultimately limit the electrical activation and the position of the introduced dopants. However, the situation is even more complex under non-equilibrium conditions as occurs during dopant implantation. The implantation process generates damage in the Si lattice that can deteriorate the performance of electronic devices. At the same time, the implanted dopants generally reside in non-substitutional positions, and thus, are electrically inactive. This makes necessary a postimplant annealing to annihilate damage and to electrically activate dopants. During these processes dopantdefect interactions take place, which enhance diffusivity above equilibrium values and decrease electrical activation through the formation of electrically inactive dopant-defect complexes. These features complicate the formation of electrically active and shallow dopant profiles. For all these reasons, B-defect interactions in $\mathrm{Si}$ has attracted much attention during the last decades. Actually, the problematic of B is still studied since next technological designs scheduled by the International Technology Roadmap for Semiconductors (ITRS) require dopant diffusion and electrical activation to be controlled under really severe processing conditions for the formation of ultra-shallow, abrupt and highly activated source and drain extensions [3]. 


\subsection{Boron diffusion in $\mathrm{Si}$}

The many studies devoted to the understanding and control of TED were correlated to the release of $\mathrm{Si}$ self-interstitials from small clusters and extended defects resulting from ion implantation [27, 25, 108]. Moreover, it has been observed experimentally that B diffusion is retarded during nitridation anneals (vacancy injection) and enhanced during oxidation anneals ( $\mathrm{Si}$ self-interstial injection) compared to reference anneals in inert ambient [126]. Thus, it has been determined that $\mathrm{B}$ diffusion in $\mathrm{Si}$ is dominated to a degree of more than $98 \%$ by an interstitial-mediated mechanism [127, 126]. The possible mechanisms for interstitial-mediated $\mathrm{B}$ diffusion are the kick-out and the interstitialcy reactions, where the interaction between a substitutional B and a self-interstitial leads to a mobile species formed by an interstitial $\mathrm{B}\left(\mathrm{B}_{i}\right)$ or a $\mathrm{B} I$ couple, respectively. From an experimental point of view, it is hard to distinguish between them, since no difference are caused in the diffused profile. Some early theoretical investigations of the energetics of $\mathrm{B}$ diffusion revealed that the preferred migration pathway is the kick-out process, so that the diffusing species is the interstitial B atom $[128,54]$. More recently, advanced DFT calculations gave a new vision of the $\mathrm{B}$ diffusion mechanism, showing that the intermediate mobile species is not the interstitial B but the BI pair $[129,130]$. Thus, these studies suggested an interstitialcy mechanism for B diffusion in which $\mathrm{B} I$ pairs diffuse in the Si lattice with an energy barrier around $0.3-0.7 \mathrm{eV}$ (close to $0.6 \mathrm{eV}$, as measured by Watkins et al. [131]) and an overall activation energy of 3.0-3.6 eV, depending on the Fermi-level position $[129,130,132]$.

This mechanism for B diffusion has been experimentally confirmed by De Salvador et al. [133]. Fig. 8 provides an overall vision of the $\mathrm{B}$ diffusion energetics in cSi reported by these authors [133-135]. The microscopic pathways of $B$ migration and the diffusivity values significantly change depending on the Fermi level position. Substitutional $\mathrm{B}\left(\mathrm{B}_{S}^{-}\right)$can give mobile $\mathrm{B} I$ pairs by interaction with neutral self-interstitials $\left(I^{0}\right)$ under intrinsic conditions or by interaction with doubly positively charged self-interstitials $\left(I^{++}\right)$under extrinsic conditions ( $p$-type doping), with energy barriers of 4.1 and $4.4 \mathrm{eV}$, respectively. In both the cases, a charge exchange with the surrounding matrix occurs giving the neutral $\mathrm{B} I^{0}$ pair, which is the migrating species. This diffusion process occurs with an energy cost of around $3.45 \mathrm{eV}$ which is the difference between the energy of the saddle point of diffusion of the $\mathrm{B} I^{0}$ species and the energy of the substitutional B atom. Instead, under high $n$-type doping conditions, the $\mathrm{B} I$ species moves

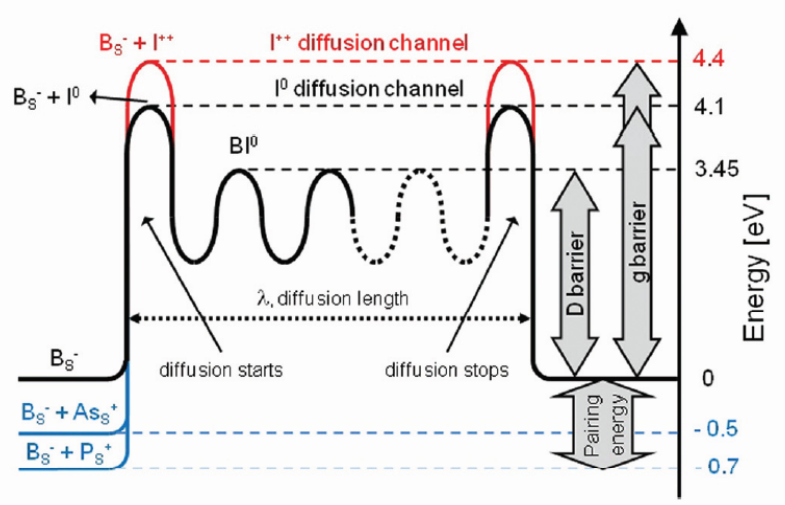

Fig. 8 Energetics of B diffusion in c-Si. The basic diffusion process occurs with an energy cost $(3.45 \mathrm{eV})$ that represents the difference between the energy of the saddle point of diffusion of the main migrating species $\mathrm{B} I^{0}$ and the energy of the substitutional $\mathrm{B}$ atom. The $\mathrm{B} I$ species moves through $\mathrm{B} I^{-}$ pairs (not shown) only under $n$-type conditions. The pairing of substitutional B with As or $\mathrm{P} n$-dopant is also indicated, causing a strong increase in the diffusion energy cost. Taken from Ref. [135].

through the negatively charged $\mathrm{B} I$ complex $\left(\mathrm{B} I^{-}\right)$. The introduction of $n$-type dopants lowers the starting energy level of substitutional B by Coulomb pairing effects of $\mathrm{B}$ with $n$-type dopants (more effective with $\mathrm{P}$ than with As), which force a fraction of $\mathrm{B}$ atoms to have a much higher-energy cost for diffusion. In any cases, $\mathrm{B}$ diffusion in crystaline $\mathrm{Si}$ is also heavily affected by nonequilibrium clustering of B atoms with Si interstitials, the so-called BICs (see section 3.2). In fact, the temporary immobilization of B into BICs alters both the density of diffusing B atoms and that of Si interstitials, needed to start the B diffusion itself.

$\mathrm{B}$ diffusion in a-Si presents different properties than those in c-Si, since it continuously changes upon annealing. Comprehension and control of this phenomenon is also relevant for technological applications since B is often introduced in preamorphized $\mathrm{Si}$, and changes in the B profiles before the complete regrowth of the preamorphized layer will significantly affect the final electrical properties of the devices [136]. Venezia et al. evidenced how $\mathrm{B}$ diffusion occurs in a-Si up to very high B concentrations $\left(\sim 2 \times 10^{20} \mathrm{~cm}^{-3}\right)$ with an activation energy of $2.1 \mathrm{eV}$ [137]. Further, a quick formation of immobile B clusters occurs during low temperature annealing $\left(500-600^{\circ} \mathrm{C}\right)$ in a-Si at concentration exceeding $\sim 2 \times 10^{20} \mathrm{~cm}^{-3}[137,138]$, in agreeement with theoretical predictions [139]. The diffusion of B in a-Si has been shown to be peculiarly transient and B concentration dependent $[137,140]$, thus it does not obey a standard Fick's law with constant and homogeneous diffusivity. Based on such a scenario, Mirabella et al. recently pro- 
posed a model that introduces a new set of defects into a-Si that can be represented by threefold-coordinated Si atoms, called dangling bonds (DBs), and fivefoldcoordinated Si atoms, called floating bonds (FBs) [140]. The proposed model explains both the peculiar concentration dependence and the transient behavior of $\mathrm{B}$ diffusion. The non-Fick-like diffusion is explained considering that the higher the B density, the more DB are present, promoting a faster $\mathrm{B}$ diffusion. The transient diffusion is related to two distinct causes for DB density reduction. The first one is the DB-FB annihilation, which quickly reduces the $\mathrm{B}$ diffusivity in the early stages of annealing. The second cause is the progressive reduction of DB density due to DB diffusion itself. These authors proposed for DB and B diffusivities activation energies of 2.6 and $3.0 \mathrm{eV}$, respectively. Recently, such a microscopic picture has been used by Martin-Bragado and Zographos to implement a KMC model for B diffusion in a-Si [141]. This model successfully simulated B diffusion in a-Si under different conditions such us molecular beam epitaxy (MBE) grown $\mathrm{B}$ marker layers is a-Si and ultrashallow B implanted into preamorphized Si.

\subsection{Boron-interstitial clusters (BICs) in $\mathrm{Si}$}

The evidence of B precipitation well below the equilibrium solid solubility in c-Si has been reported by many authors during the last decades together with the early TED studies of B implanted in Si $[26,41,142,29,15]$. Thermal annealing after B implantation typically leads to a peak portion of the $\mathrm{B}$ profile, which is immobile and electrically inactive, whereas a lower part of the profile undergoes TED, with a concentration threshold about one order of magnitude below the B solid solubility $[26,142]$. It was proposed that the process responsible for the non equilibrium B precipitation through BICs formation was the same responsible for the TED effect, i.e., the Si interstitial supersaturation [142]. Further confirmation of this came with the evidence that B clustering effectively contributes to lower the Si interstitial supersaturation following ion implantation [143]. In addition, a proper substitutional B concentration was demonstrated to suppress the typical $\{113\}$ defects, by a competitive BIC formation [124]. Therefore, there is a consensus on the idea that B clustering is associated to the interaction between several B atoms and Si selfinterstitials to form immobile and electrically inactive BICs. Obviously, this phenomenon has a clear negative technological drawback as far as the dopant activation is concerned. In addition, it also affects B diffusion since $\mathrm{B}$ atoms are temporarily immobilized and Si interstitial supersaturation is modified during BIC formation and dissolution. Therefore, the knowledge of the BICs features is also essential to develop a complete simulation model of the B diffusion in Si.

The phenomena associated to B clustering in c-Si have been analyzed by several authors from experimental $[113,144,115]$ and theoretical [145-151] points of view, determining the total energy of each plausible $\mathrm{B}_{n} I_{m}$ configuration (complex with $n \mathrm{~B}$ atoms and $m$ Si interstitials) and the possible pathways for BIC growth and dissolution. Up to a few years ago, BICs were undetectable by TEM, thus models only considered BICs of small size, typically containing less than 10 atoms (generally up to $4 \mathrm{~B}$ atoms and several Si interstitials), well below the TEM detection limit. In all those models the kinetics of $\mathrm{B}$ deactivation/activation is controlled by the growth/dissolution of the $\mathrm{B}_{n} I_{m}$ complexes through the trapping/emission of mobile species $(\mathrm{B} I, I)$, described by the following reactions:

$\mathrm{B}_{n} I_{m}+\mathrm{B} I \leftrightarrow \mathrm{B}_{n+1} I_{m+1}$

$\mathrm{B}_{n} I_{m}+I \leftrightarrow \mathrm{B}_{n} I_{m+1}$

Based on these reactions, adding a $\mathrm{B} I$ pair or an I to a preexisting $\mathrm{B}_{n} I_{m}$ cluster entails a reduction in the energy of the overall system given by the following expressions, respectively:

$$
\begin{aligned}
E_{b i n d}^{B I}\left(\mathrm{~B}_{n+1} I_{m+1}\right)= & E_{t o t}\left(\mathrm{~B}_{n} I_{m}\right)+E_{t o t}(\mathrm{~B} I)- \\
& E_{t o t}\left(\mathrm{~B}_{n+1} I_{m+1}\right)
\end{aligned}
$$

$$
\begin{aligned}
E_{b i n d}^{I}\left(\mathrm{~B}_{n} I_{m+1}\right)= & E_{t o t}\left(\mathrm{~B}_{n} I_{m}\right)+E_{t o t}(I)- \\
& E_{t o t}\left(\mathrm{~B}_{n} I_{m+1}\right)
\end{aligned}
$$

where $E_{\text {tot }}$ represents the total energy of each particular defect whereas $E_{b i n d}^{B I}$ and $E_{b i n d}^{I}$ are the binding energies of $\mathrm{B} I$ and $I$ to the particular BIC configuration, respectively. Total energies for defects could be referred to different ground levels. Some authors considered as the reference level the energy of free B atoms and free Si interstitials, so free B and free Si interstitials have assigned 0 energy. Alternatively, the perfect lattice could be considered as the reference level for the definition of total energies. Depending on the considered reference level the values for $E_{t o t}(B I)$ and $E_{t o t}(I)$ that should be included in Eqs. 3-4 are diferent. In the first case, $E_{\text {tot }}(B I)$ and $E_{\text {tot }}(I)$ are the binding energy of the $\mathrm{B} I$ pair and null energy, respectively, whereas in the last case, these values reprensent respectively the formation energy of free Si intersititial and the formation energy of $\mathrm{B} I$ from the perfect lattice (i.e., the total energy of $\mathrm{B}_{n} I_{m}$ with $n=m=1$, referred to the perfect lattice). Independently of the reference level, negative values for binding energies of Eqs. 3-4 indicate that a barrier for 
the trapping of the particular mobile specie from the $\mathrm{B}_{n} I_{m}$ cluster exists. In contrast, positive values indicate that the trapping of the particular mobile specie is energetically favorable, and the higher is the energy value the more stable the resulting BIC is. Thus, the possible pathways for BIC growth and dissolution are determined by total energies of BICs. Different authors reported different values for total energies for BICs, although most of them agree on the overall description of the system.

Generally, models for BICs considered that large BICs were not energetically favorable compared to those with 3-4 B atoms since they were no detectable by TEM. Nevertheless, recently some studies have evidenced that BICs as large as a few nanometers could form under particular conditions of very high concentrations (as those required as devices are aggressively scaled down). In particular, weak beam-dark-field (WBDF) analyses performed on high-dose, $0.5 \mathrm{keV} \mathrm{B}$ implanted c-Si, after a low-temperature annealing, evidenced the formation of large BICs (with hundreds of atoms) within the damage region [152]. Later on, large BICs have been observed also out of the implant damage region, by employing an MBE grown sample (containing a buried, highly doped B profile) subjected to shallow Si implantation [153]. In this last case, the immobile B peak deduced from chemical profiling was found to overlap with the band of BICs observed by TEM. Moreover, in this last case it was demonstrated that BICs clearly showed two different paths for dissolution with different energy barriers (3.6 or $4.8 \mathrm{eV}$ ) and dissolution rates. The comparison of BIC dissolution rates with TEM showed that the faster path was associated to the dissolution of small BICs (not visible by TEM) whereas the slower path was connected to the dissolution of observable large BICs (only present for very high B concentrations) [153,154].

Based on these evidences, recently we have developed a comprehensive model for BIC formation and evolution in c-Si, including $\mathrm{B}_{n} I_{m}$ complexes quite larger than those included in previous models [115]. BIC evolution is modeled as drawn in Fig. 9, considering exchange of B (oblique red lines) or Si interstitials (vertical blue lines) with the hosting lattice to change BIC configuration, and taking the total energy of each $\mathrm{B}_{n} I_{m}$ cluster (referred to the perfect lattice) through comparison with theoretical calculations and fitting models to different sets of experimental data. In Fig. 9, four main regions have been defined, one region (SB, small BICs) for clusters with less than $4 \mathrm{~B}$ atoms (typical small configurations included in previous models), and three regions for large BICs (LBLI-large BICs low interstitial, LBB-large BICs barrier, and LBHI-large BICs high interstitial) with a larger number of $\mathrm{B}$ atoms. The schematic placed in the lower panel of Fig. 9 summarizes the main characteristics of the four different regions considered in the model. Gray and white arrows distinguish between growth and dissolution paths, respectively, whereas solid and dashed arrows indicate high and low probability paths, respectively. SB region includes small BICs $(n<4)$ that reproduce experimental data at low and medium B concentrations. LBLI region considers very stable large BICs $(n>4$, $m<<n$ ) that form from SB region and only in the presence of high $\mathrm{B}$ concentration and low flux of interstitials (their dissolution requires very intense thermal budgets). LBHI region with less stable large BICs ( $n>4$ and large amount of interstitials) coming from $\mathrm{SB}$ region if high $\mathrm{B}$ concentration and high flux of interstitials subsist (but they could easily evolve toward BICs of SB region through BI emission at relatively low thermal budgets). Finally, LBB region represents quite unstable large BICs $(n>4$ and intermediate amount of interstitials) that act as a barrier among less stable and very stable large BICs.

Finally, some considerations should be done on B clustering in a-Si. The use of preamorphized Si samples followed by thermal induced SPER is increasingly more demanded for the fabrication of ultrashallow and extremely highly doped junctions. The recrystallization of the a-layer eliminates the implant damage thus avoiding the overlapping of excess Si interstials and B atoms. Nevertheless, it has been shown that B clustering already occurs in preamorphized $\mathrm{Si}$, even if to a lower extent than in c-Si. Thus, Venezia et al. evidencing how during low temperature annealing $\left(500-600^{\circ} \mathrm{C}\right)$ $\mathrm{B}$ diffusion occurs in a-Si up to very high $\mathrm{B}$ concentrations $\left(\sim 2 \times 10^{20} \mathrm{~cm}^{-3}\right)$, at least two orders of magnitude higher than the mobile $\mathrm{B}$ concentration in c-Si at the same temperatures [137]. However, B profiles still evidence an immobilization of $\mathrm{B}$ atoms for concentrations above $\sim 2 \times 10^{20} \mathrm{~cm}^{-3}[34,35,137]$ which has been attributed to B clustering in a-Si that electrically deactivates $B$ atoms. Actually, sheet resistance measurements immediately after SPER are consistent with maximum levels of $\mathrm{B}$ activation of around $\sim 2 \times 10^{20} \mathrm{~cm}^{-3}[34,35$, 155]. The modeling of BICs in a-Si is more complex and the energetics may not be the same as in c-Si. In fact, the kinetics of BICs in a-Si has not been modelled in detail yet. In turn, it is usually assumed as the initial conditions after SPER that BICs appear above $2 \times 10^{20}$ $\mathrm{cm}^{-3}$ in the most stable configurations compatible with the energetics included in Fig. 9 [115]. By using MD calculations, Mattoni and Colombo have characterized the formation kinetics of some BICs during recrystallization and found that $\mathrm{B}_{2} I, \mathrm{~B}_{3} I$ and $\mathrm{B}_{4} I_{2}$ configurations could play the major role in the evolution of re- 


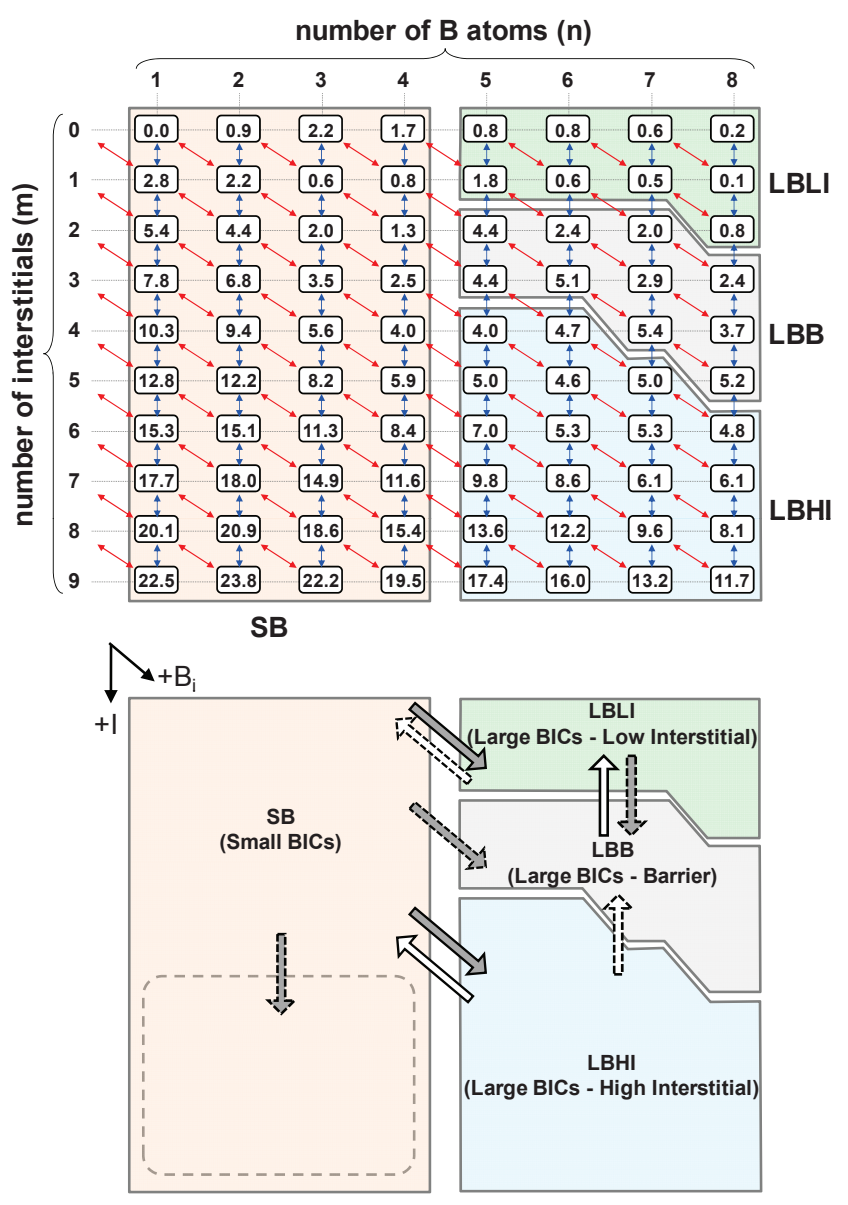

Fig. 9 Schematic representations of (upper panel) the energetics of the several BIC configurations $\left(\mathrm{B}_{n} I_{m}\right)$ referred to the perfect lattice and (lower panel) the main features of the proposed model which includes four main regions. In the upper panel oblique (red) and vertical (blue) arrows represent the formation/dissolution paths for the different configurations through the trapping/emission of mobile species boron-interstitial $(\mathrm{B} I)$ and $\mathrm{Si}$ interstitial $(I)$, respectively. In the lower panel grey and white arrows distinguish between formation and dissolution paths, respectively, whereas solid and dashed arrows indicate high and low probability paths, respectively.

crystallized B-doped Si [139]. Recently, by using KMC simulations combined with experimental data we have proposed $\mathrm{B}_{2}, \mathrm{~B}_{3} I, \mathrm{~B}_{4} I$ and $\mathrm{B}_{4} I_{2}$ small BIC configurations as the more probable and energetically favored BICs after recrystallization, with ratios that depend on B concentration [115]. Note that such BICs, even if formed in preamorphized $\mathrm{Si}$, are transferred to the c-Si once the SPER is complete. Thus, their posterior evolution during subsequent device fabrication process steps takes place in c-Si, and thus, it is modeled according to energetics included in Fig. 9.

In summary, the non equilibrium clustering of B induced by a supersaturation of Si interstitials leads to

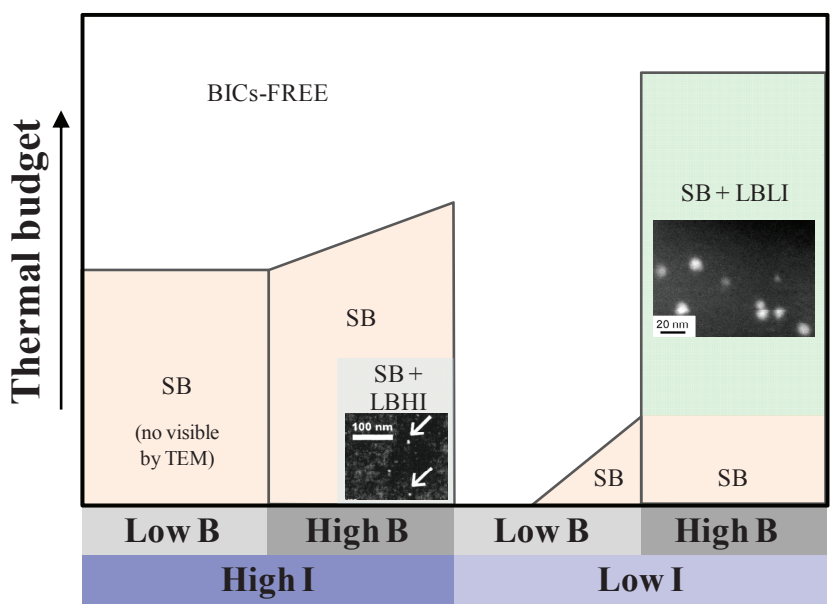

Fig. 10 Schematic diagram of the behavior of BICs as a function of the starting conditions (B concentration and flux of Si interstitials) and thermal budget. SB, LBHI and LBLI refer to the different BIC regions modeled in Fig. 9. TEM images examples of observable large BICs are taken from Refs. $[152,115]$.

$\mathrm{B}_{n} I_{m}$ complexes (named BICs), whose size and thermal stability depend on the starting conditions. Figure 10 gives a schematic diagram for BICs behavior as a function of the starting conditions and thermal budget, based on experimental observations and KMC simulations (see Ref. [115] and references therein). In this diagram we distinguish between "Low B" or "High B", which means that the $\mathrm{B}$ profile has a peak concentration lower or higher than $\sim 10^{20} \mathrm{~cm}^{-3}$, respectively. We also consider different situations for Si interstitials, in particular:

- "High I" refers to situations in which BICs evolution occurs in the presence of a high flux of Si interstials. This occurs, for instance, when B is implanted in c-Si since the high concentration of Si intersititials that results from the implantation process overlaps with the implanted B profile. Under these conditions, B electrical deactivation and precipitation well below the equilibrium solid solubility has been evidenced for both low and high B concentrations, which has been associated to the formation of BICs $[26,124,156,152]$. According to the developed model (Fig. 9), for low and medium B concentration profiles only BICs contained in SB region are formed. Adding Si interstitials the stability of these BICs is increased, at least up to configurations with around 1.2 Si interstitials per B atom, in agreement with experimental data [157]. Note that small BICs with large amount of Si interstitials (dashed region in Fig. 9(b)) have very high energy in the model and thus, are quite unstable and have very low probability to form. In the presence of very high B concentrations 
$\left(\sim 10^{21} \mathrm{~cm}^{-3}\right)$ the evolution of BICs into configurations as large as $10 \mathrm{~nm}$ has been reported by weakbeam-dark-field (WBDF) TEM investigations [152] (modeled as HBHI region in Fig. 9). These BICs require relatively low thermal budget to become "invisible" by TEM although BICs are still detected by Secondary Ion Mass Spectroscopy (SIMS) measurements at high temperature, indicating that their size probably decreases below the TEM detection limit. This is modeled by considering BICs of HBHI region as not very stable stable configurations that quickly return to SB configurations with relatively low thermal budget.

- "Low I" makes reference to situations in which the flux of interstitials is low, as occurs for instance when B is implanted in preamorphized Si. Under these conditions, only EOR interstitial defects beyond the a/c interface (typically deeper than the B profile) remain after regrowth $[23,24]$. Thus, as $\mathrm{Si}$ interstitial defects do not overlap with the B profile, the flux of Si interstitials coming from the EOR defects towards the B profile is low. For low B concentrations (lower than $\sim 10^{20} \mathrm{~cm}^{-3}$ ) the formation of BICs is not possible if the flux of Si interstitials is very low, in agreement with experimental data reported by Jones et al. [158]. For higher B concentrations, when SPER experiments are concerned, small BICs formation occurs even during the recrystallization itself, as confirmed by experimental data and theoretical calculations $[34,35,139]$. Additionally, during thermal proccesing after recrystallization preexisting BICs evolve in recrystallized $\mathrm{Si}$, thus being governed by energetics of Fig. 9. These preexisting small can evolve towards BICs as large as $8 \mathrm{~nm}$ if a high $\mathrm{B}$ concentration is present and subjected to a proper low Si interstitial supersaturation (modeled as HBLI region in Fig. 9), as shown by $\mathrm{B}$ chemical mapping and energy filtered TEM (EFTEM) investigations $[153,115]$. These large BICs have shown high stability since they need very intense thermal budgets to dissove.

\subsection{Applications of BICs modeling to physical understanding and technology}

In this subsection we present some examples of KMC simulations based on experiments in which B diffusion and clustering processes are involved. According to the most commonly accepted theoretical calculations, we consider that B diffuses by an interstitialcy mechanism, based in $\mathrm{B}$ diffusion through a mobile $\mathrm{B} I$ pair with migration and binding energies taken from Ref. [129]. The precipitation and immobilization of $\mathrm{B}$ atoms is modeled by the formation of electrically inactive BICs whose evolution is controlled by energetics included in Fig. 9.

\subsubsection{Kinetics of BICs under low Si interstitial flux: evidence of two dissolving species of BICs}

The BICs model shown in Fig. 9 has been successfully applied to BICs evolution in both crystalline and preamorphized Si samples [115]. As an example, Fig. 11 shows experimental data (reported by De Salvador et al. [154]) and simulation results for the dissolution kinetics of BICs formed in c-Si by employing MBE grown samples (containing a buried doped B profile at concentrations either below and above equilibrium solid solubility). These samples were subjected to shallow $\mathrm{Si}$ implantation (20 keV Si ions - projected range around $30 \mathrm{~nm}$ - at a nonamorphizing dose of $1 \times 10^{14} \mathrm{~cm}^{-2}$ ) that generates a band of defects close to the Si surface which does not overlap with the B profile, as it is schematically shown in the inset of Fig. 11(a). Similarly to experiments, for KMC simulations fully substutional box-shaped B profiles at concentrations below solid solubility $\left(10^{19} \mathrm{~cm}^{-3} \mathrm{~B}\right.$ box, sample A - at $815^{\circ} \mathrm{C}$ solid solubility is $\sim 3.8 \times 10^{19} \mathrm{~cm}^{-3}$ [159]) and well above solid solubility $\left(2 \times 10^{20} \mathrm{~cm}^{-3} \mathrm{~B}\right.$ box, sample B) and at a depth of $220 \mathrm{~nm}$ were considered. After the Si implant, a rapid thermal annealing at $815^{\circ} \mathrm{C}$ for 5 min was performed. Simulations show that this thermal process fully dissolves the implant induced defects and injects Si interstitials towards the B profile. According to previous work, under these conditions in which the $\mathrm{B}$ profile do not overlap the damaged region, only a small fraction of the total B atoms evolve to BICs and the low flux of intersitials is not enough to form BICs with a high Si interstitial content [39]. Later, additional anneals at $900^{\circ} \mathrm{C}$ were performed in both samples in order to analyze the evolution of the clustered B dose. In Fig. 11(a) we represent reported experimental data [154] for the evolution of the clustered $\mathrm{B}$ dose during annealing at $900^{\circ} \mathrm{C}$ along with our simulation results obtained by only considering BICs of SB region included in Fig. 9 as stable configurations (BICs with $n>4$ were considered very unstable, similar to other proposed models [145-151]). As discussed in Ref. [154], experimental data show that BICs dissolution in sample A shows only a fast dissolution rate whereas sample B presents two stages in the dissolution, firstly a fast and later a slow dissolution rate. Our model without large BICs reproduces experimental data for sample A. Nonetheless, in the case of sample B simulation results in a lower dose of clustered B than expected and does not predict the slow dissolution rate. In contrast, our extended model for B 
clustering shown in Fig. 9, including large BICs $(n>4)$, allows us to reproduce experimental data for both samples as can be seen in Fig. 11(b). We also include in this figure the dose of large BICs formed during annealing of sample B. Note that for sample A the result of the simulation is identical to the one obtained with the model without large BICs (see Fig. 11(a)). Our simulations show that no large BICs are formed in sample A, due to the low B concentration, and thus the decrease of the clustered dose is only controlled by the dissolution of small BICs. In the case of sample B, simulations show that injected Si interstitials during the first annealing step at $815^{\circ} \mathrm{C}$ lead to the growth of small BICs of SB region, but also a fraction of them (around $30 \%$ of total BICs) evolves from SB region towards larger and more stable BICs of LBLI region (mainly in the form of $\mathrm{B}_{n}$ and $\mathrm{B}_{n} I$ configurations). During the second step anneal at $900^{\circ} \mathrm{C}$, initially small BICs of SB region start to dissolve by emission of $\mathrm{B} I$ (with an activation energy $\sim 3.7 \mathrm{eV}$ ) whereas the dose of large BICs remains almost constant. Once small BICs of SB region fully dissolve (after $\sim 1500 \mathrm{~s}$ anneal) the dissolution rate significantly decreases as it is only controlled by the emission of $\mathrm{B} I$ from large and more stable BICs of LBLI region (with an activation energy $\sim 4.8 \mathrm{eV}$ ). Thus, even if the largest BICs that can be included in our model contain less than 20 atoms, two different dissolution pathways have been found according to experiments, a faster one for BICs of SB region and a slower one for BICs of LBLI region.

\subsubsection{Analysis of implanted B emitters for solar cell applications}

N-type Si wafers for solar cells have received considerable attention recently due to their electrically superior properties compared to p-type $\mathrm{Si}$, such as higher tolerance to metallic impurities, much better stability under illumination, and higher bulk lifetime [160-163, $5,164]$. In spite of these advantages, n-type Si wafers are not widely used in mainstream solar-cell production due to the complexity of B-doped emitter formation and its passivation for mass production [165]. Typically, B doped emitters are industrially realized by diffusion from a solid, vapor, or liquid source. However, recently ion implantation has gained more attention as a potential alternative for the fabrication of Si solar cells due to the expected ease of automation [2]. Also, the experience gathered from CMOS processing enables excellent profile engineering, with independent control over the peak surface doping concentration and the doping depth even for high sheet resistances, which result in increased throughput and improved cell performance.
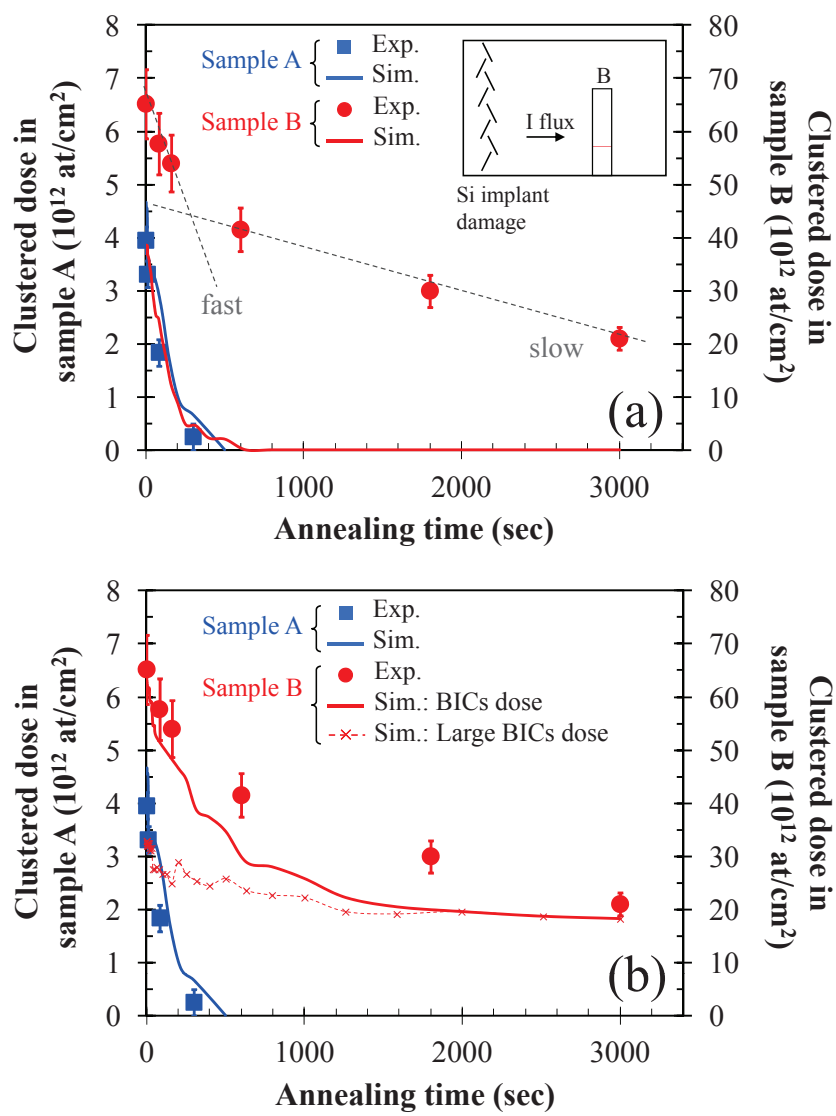

Fig. 11 Experimental data (symbols) [154] and simulation results (lines) for the evolution of the clustered $\mathrm{B}$ dose as a function of annealing time at $900 \mathrm{C}$ for samples A $\left(10^{19}\right.$ $\mathrm{cm}^{-3} \mathrm{~B}$ box $)$ and $\mathrm{B}\left(2 \times 10^{20} \mathrm{~cm}^{-3} \mathrm{~B}\right.$ box $)$ implanted with $\mathrm{Si}$ ions at $20 \mathrm{keV}, 1 \times 10^{14} \mathrm{~cm}^{-2}$. (a) Simulations performed by considering a classical $\mathrm{B}$ clustering model are not able to reproduce the evolution of sample $B$ with two different dissolution paths (dashed lines are a fit to the experimental data by the sum of two exponential decays, firstly faster and later slower). A schematic of the experiment is shown in the inset. (b) The extended model for BICs shown in Fig. 9, which includes very stable BICs with more than $4 \mathrm{~B}$ atoms (larger than in classical models), allow us to capture the two different regimes of dissolution. The dose of such large and very stable $\mathrm{BICs}$ is also included in the figure.

The requirements for B doped emitter formation in solar cells are mainly related to crystal purity that enables long minority carrier lifetimes, medium B doping levels for good conduction, and contacting properties. Here we analyze by KMC simulations a recently reported experimental study on B emitters of solar cells fabricated by B implantation in c-Si followed by hightemperature anneal to electrically activate B atoms [166]. In those experiments different $\mathrm{B}$ emitters were realized by B implantation at the rear surface of the solar cells with a fixed energy of $5 \mathrm{keV}$ and variable doses ranging from $1 \times 10^{14}$ to $3 \times 10^{15} \mathrm{~cm}^{-2}$ at room temperature. Post-implant thermal processing at $900^{\circ} \mathrm{C}$ for 2 min was 
performed in oxidizing ambient for good front and rear passivation and followed by $1000^{\circ} \mathrm{C}$ for $10 \mathrm{~min}$ in $\mathrm{N}_{2}$ ambient to obtain a good activation of dopants. Fig. 12 plots the experimental and simulated values for the sheet resistance $R_{s}$ of the $\mathrm{B}$ emitters along with the simulation results for the dose of $\mathrm{B}$ atoms stored in BICs resulting after the B implant and thermal processes. Simulations shows that $R_{s}$ decreases with increasing implant B dose, in very good agreement with experimental data. Thus, an improvement in the performance of the solar cell as B dose increases could be expected. However, some parameters of the solar cell such us the open-circuit voltage, $V_{o c}$, did not show this trend, as it is shown in inset of Fig. 12. Experiments revealed that $V_{o c}$ increases up to B implant dose of $5 \times 10^{14}$ but decreases for higher implant doses, due to a significant drop in the minority carrier effective lifetime. Simulations show that lifetime degradation could be associated to the presence of BICs. Under these experimental conditions the situation is quite different from the one previously analyzed since the highly damaged region ( $\mathrm{Si}$ interstitials and vacancies) resulting from the B implant overlaps with the implanted B profile. Simulations show that during the B implant itself a significant dose of B is immobilized in small BICs ( $\mathrm{SB}$ region) with a high Si interstitial content (around 1.2-1.5 interstitials per $\mathrm{B}$ atom), according to previous experimental and simulation works $[157,39,115]$. As it is shown in Fig. 12, after the thermal processes these BICs are able to fully dissolve if $\mathrm{B}$ implant doses up to $5 \times 10^{14}$ are considered. In contrast, if higher B implant doses are used, a significant dose of B atoms still remain stored in BICs. These defects could be responsible for the minority carrier lifetime degradation observed in experiments.

\section{Conclusions}

In this article we have shown how atomistic simulation techniques can be used to develop Si-processing models with predictive capabilities. The fabrication of small Si devices brings up complex physical mechanisms, whose modeling requires a multiscale approach. Atomistic methods such as ab initio or MD can provide the mechanisms and parameters that describe the physics of the system. To reach macroscopic scales simplified models based on the physics provided by the atomistic calculations need to be performed. KMC methods can be used to define the range of validity of some approximations and also can be directly applied in process simulators of nanometer devices.

Ion implantation continues as the most promising technique to introduce dopants in Si substrates. In this

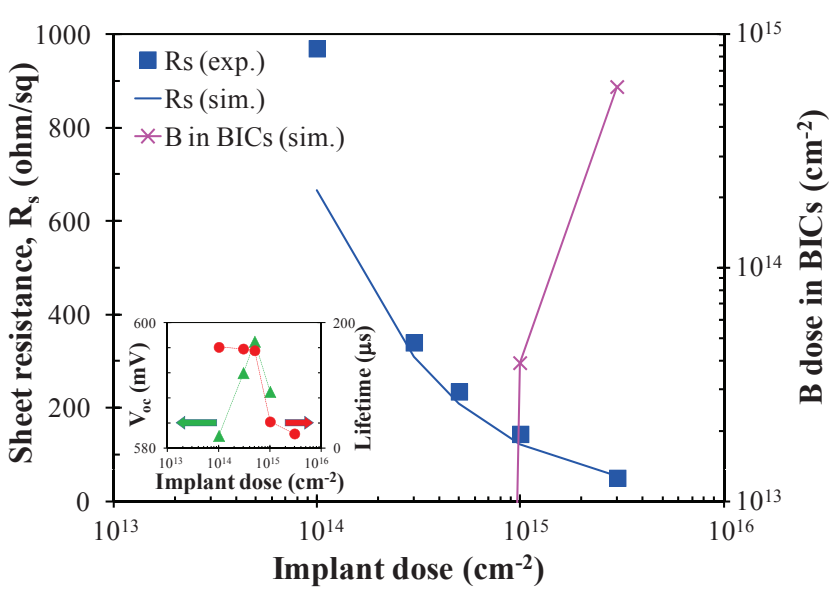

Fig. 12 Experimental (symbols) [166] and simulated values for $R_{s}$ of $\mathrm{B}$ emitters of solar cells fabricated by B implantation with energy of $5 \mathrm{keV}$ at different doses. Post-implant thermal processing at $900^{\circ} \mathrm{C}$ for $2 \mathrm{~min}$ in oxidizing ambient followed by $1000^{\circ} \mathrm{C}$ for $10 \mathrm{~min}$ in $\mathrm{N}_{2}$ ambient was performed. The simulation results for the dose of $\mathrm{B}$ atoms stored in BICs resulting after the $\mathrm{B}$ implant and thermal processes is also included. The inset includes experimental data for $V_{o c}$ and minority carrier effective lifetime [166]. Simulations suggest that the presence of BICs for high implant doses could be responsible for the minority carrier lifetime degradation and the resulting $V_{o c}$ decrease observed in experiments.

article we reviewed the key features of models for defects resulting from ion implantation and interactions between $\mathrm{B}$ and defects. These models need to be accurate in order to describe the kinetics of damage as well as B migration and clustering in Si. We also identified the type of defects that are predominant depending on the experimental parameters. We illustrated with some examples how the accuracy of models could be crucial for some type of simulations or, in turn, the use of simplified models could be enough to perform predictive simulations.

The morphology of the damage produced by irradiation spans from point defects to small clusters and extended defects, which requires an appropriated model for each type of defect. For very low implant doses, as those used in PL applications, only small defects are formed. Theoretical calculations give a diversity of results for such small defects, and in particular, for Si interstitial clusters there are significant discrepancies. We found that simulation results are very sensitive to the model used for small Si interstitial clusters which complicates the extraction of reliable conclusions from simulations. Nevertheless, we found that for medium and high implant doses (typically required in junction formation for ICs fabrication) the model for small Si interstitial clusters is no so relevant. At this regime, small Si interstitial defects quickly evolve to extended defects (whose models are more clearly established and 
accepted by the material science community). Extended defects survive for much longer time until they are annihilated at the Si surface, and macroscopic observations associated to defects (such us TED) are mainly controlled by the evolution of the extended defects.

In c-Si, B diffusion takes place through an interstitialcy mechanism, being the neutral $\mathrm{B} I_{0}$ pair the main migrating species. Thus, B diffusion is enhanced if a large supersaturation of Si interstitials exists. Furthermore, B diffusion in c-Si is also heavily affected by the formation of BICs that temporarily immobilize B atoms altering both the density of diffusing $\mathrm{B}$ atoms and $\mathrm{Si}$ interstitials. In a-Si, B diffusivity is quite larger than in c-Si, causing significant broadening of B profiles as well as a very quick B precipitation when B is implanted in preamorphized Si during ultra-shallow junction fabrication. These precipitates are transferred to c-Si once the a-layer recrystallizes, thus behave as BICs in c-Si. In any cases, BICs affect the electrical behavior because of $\mathrm{B}$ deactivation and eventually charge carrier mobility degradation [115]. They also affects carrier effective lifetime which can degrade the efficiency of solar cells. Under prolonged annealing, these BICs dissolve releasing both B and Si interstitials. BICs are usually very small (below $1 \mathrm{~nm}$ in size) and dissolve with an activation energy of $3.7 \mathrm{eV}$. Such BICs were included in classical models for BICs, being these simplified models appropriated to simulate experiments in which B is implanted at low and medium doses. However, in applications in which very large $B$ concentrations are present (as those obtained by ultra-low energy and high-dose B implants required in the fabrication of ultra-shallow junctions) the evolution from small BICs into quite large configurations (5-10 $\mathrm{nm}$ in size) is possible. Such large configurations could be very stable under conditions of very high B concentrations and low Si interstitial supersaturation, dissolving with an activation energy of $4.8 \mathrm{eV}$ (larger than for small BICs). Thus, under these high B concentration conditions, the extended model for BICs reported in this work is required.

Acknowledgements We want to acknowledge funding from the Spanish Goverment under project TEC2011-27701.

\section{References}

1. E. Chason, S.T. Picraux, J.M. Poate, J.O. Borland, M.I. Current, T.D. de la Rubia, D.J. Eaglesham, O.W. Holland, M.E. Law, C.W. Magee, J.W. Mayer, J. Melngailis, A.F. Tasch, J. Appl. Phys. 81, 6513 (1997)

2. A. Rohatgi, D. Meier, B. McPherson, Y.W. Ok, A. Upadhyaya, J.H. Lai, F. Zimbardi, Energy Procedia 15, $10(2012)$

3. International Technology Roadmap for Semiconductors, http://www.itrs.net/
4. J. Chen, IEDM Technical Digest p. 1 (2009)

5. J.E. Cotter, J.H. Guo, P.J. Cousins, M.D. Abbott, F.W Chen, K.C. Fisher, IEEE Trans. Electron Devices 53, 1893 (2003)

6. P. Giri, S. Coffa, E. Rimini, Appl. Phys. Lett. 78, 291 (2001)

7. J. Bao, M. Tabbal, T. Kim, S. Charnvanichborikarn, J. Williams, M. Aziz, F. Capasso, Optics Express 15, 6727 (2007)

8. FLOOPS (FLorida Object Oriented Device and Process Simulator), http://www.flooxs.tec.ufl.edu/

9. M.E. Law, S.M. Cea, Comput. Mat. Sci. 12, 289 (1998)

10. C. Rafferty, R.K. Smith, CMES-Comp. Model. Eng. 1 151 (2000)

11. T. Noda, W. Vandervorst, S. Felch, V. Parihar, C. Vranckena, S. Severi, A. Falepin, T. Janssens, H. Bender, B.V. Daele, P. Eyben, M. Niwa, R. Schreutelkamp, F. Nouri, P. Absil, M. Jurczak, K.D. Meyer, S. Biesemans, IEDM Technical Digest p. 1 (2006)

12. K.R.C. Mok, B. Colombeau, F. Benistant, R.S. Teo, S.H. Yeong, B. Yang, M. Jaraiz, S.S. Chu, IEEE Transac. Electron Devices 54, 2155 (2007)

13. M. Jaraiz, P. Castrillo, R. Pinacho, J.E. Rubio, IEDM Technical Digest p. 951 (2007)

14. L. Pelaz, R. Duffy, M. Aboy, L.A. Marqués, P. López I. Santos, B.J. Pawlak, M.J.H. van Dal, B. Duriez, T. Merelle, G. Doornbos, N. Collaert3, L. Witters, R. Rooyackers, W. Vandervorst, M. Jurczak, M. Kaiser, R.G.R. Weemaes, J.G.M. van Berkum, P. Breimer, R.J.P. Lander, in IEDM Technical Digest (2008), p. 535

15. L. Pelaz, M. Jaraiz, G.H. Gilmer, H.J. Gossmann, C.S Rafferty, D.J. Eaglesham, J.M. Poate, Appl. Phys. Lett. 70, 2285 (1997)

16. M. Jaraiz, P. Castrillo, R. Pinacho, I. Martín-Bragado, J. Barbolla, in Simulation of Semiconductor Processes and Devices, ed. by D. Tsoukalas, C. Tsamis (SpringerVerlag, Wien, 2001), p. 10

17. P. Hohenberg, W. Kohn, Phys. Rev. 136, B864 (1964)

18. W. Kohn, Rev. Mod. Phys. 71, 1253 (1999)

19. N.W. Ashcroft, N.D. Mermin, Solid State Physics (HRW International Editions, New York, 1976)

20. F. Bloch, Z. f. Physik 52, 555 (1928)

21. L. Colombo, Annu. Rev. Mater. Res. 32, 271 (2005)

22. M.D. Giles, J. Electrochem. Soc. 138, 1160 (1991)

23. A. Claverie, B. Colombeau, G.B. Assayag, C. Bonafos, F. Cristiano, M. Omri, B. de Mauduit, Mat. Sci. Semicond. Process. 3, 269 (2000)

24. A. Claverie, B. Colombeau, B.D. Mauduit, C. Bonafos, X. Hebras, G.B. Assayag, F. Cristiano, Appl. Phys. A 76, 1025 (2003)

25. N.E.B. Cowern, G. Mannino, P.A. Stolk, F. Roozeboom, H.G.A. Huizing, J.G.M. van Berkum, F. Cristiano, A. Claverie, M. Jaraiz, Phys. Rev. Lett. 82, 4460 (1999)

26. W.K. Hofker, H. Werner, D.P. Oosthoek, H.A.M. de Grefte, Appl. Phys. 2, 165 (1973)

27. D.J. Eaglesham, P.A. Stolk, H.J. Gossmann, J.M. Poate, Appl. Phys. Lett. 65, 2305 (1994)

28. P.M. Rousseau, P.B. Griffin, J.D. Plummer, Appl. Phys. Lett. 65, 578 (1994)

29. P.A. Stolk, H.J.G.D.J. Eaglesham, D.C. Jacobson, C.S. Rafferty, G.H. Gilmer, M. Jaraiz, J.M. Poate, H.S. Luftman, T.E. Haynes, J. Appl. Phys. 81, 6031 (1997)

30. S. Whelan, V. Privitera, G. Mannino, M. Italia, C. Bongiorno, A. La-Magna, E. Napolitani, J. Appl. Phys. 90, 3873 (2001) 
31. S.C. Jain, W. Schoenmaker, R. Lindsay, P.A. Stolk, S. Decoutere, M. Willander, H.E. Maes, J. Appl. Phys. 91, 8919 (2002)

32. S. Solmi, M. Ferri, M. Bersani, D. Giubertoni, V. Soncini, J. Appl. Phys. 94, 4950 (2003)

33. S. Prussin, D.I. Margolese, R.N. Tauber, J. Appl. Phys. 57, $180(1985)$

34. J.Y. Jin, J. Liu, U. Jeong, S. Mehta, K. Jones, J. Vac. Sci. Technol. B 20, 422 (2002)

35. W. Lerch, S. Paul, J. Niess, F. Cristiano, Y. Lamrani, P. Calvo, N. Cherkashin, D.F. Downey, E.A. Arevalo, in Electrochem. Soc. Proc. 2004 (2004)

36. L. Csepregi, E.F. Kennedy, J.W. Mayer, T.W. Sigmon, J. Appl. Phys. 49, 3906 (1978)

37. L. Pelaz, L.A. Marqués, J. Barbolla, J. Appl. Phys. 96, 5947 (2004)

38. K.S. Jones, K. Moller, J. Chen, M. Puga-Lambers, B. Freer, J. Berstein, L. Rubin, J. Appl. Phys. 81, 6051 (1997)

39. M. Aboy, L. Pelaz, L.A. Marqués, P. López, J. Barbolla, R. Duffy, V.C. Venezia, P.B. Griffin, Appl. Phys. Lett. 86, 031908 (2005)

40. S. Hong, G. Ruggles, J. Wortman, M. Ozturk, IEEE Trans. Elec. Dev. 38, 476 (1991)

41. P.M. Fahey, P.B. Griffin, J.D. Plummer, Rev. Mod. Phys. 61, 289 (1989)

42. T.Y. Tan, U. Gösele, Appl. Phys. A: Solids Surf. 37, 1 (1985)

43. P. Pichler, Intrinsic Point Defects, Impurities, and Their Diffusion in Silicon (Springer, Wien, 2004)

44. G.D. Watkins, J.W. Corbett, Phys. Rev. 134, A1359 (1964)

45. S. Dannefaer, P. Mascher, D. Kerr, Phys. Rev. Lett. 56, 2195 (1986)

46. H. Bracht, N.A. Stolwijk, H. Mehrer, Phys. Rev. B 52, $16542(1995)$

47. M.I.J. Probert, M.C. Payne, Phys. Rev. B 67, 075204 (2003)

48. J. Lento, R.M. Nieminen, J. Phys.: Condens. Matter 15, 4387 (2003)

49. S. Takeda, M. Kohyama, K. Ibe, Philos. Mag. A 70, 287 (1994)

50. A.E. Michel, W. Rausch, P.A. Ronsheim, R.H. Kastl, Appl. Phys. Lett. 50, 416 (1987)

51. P.E. Blöchl, E. Smargiassi, R. Car, D.B. Laks, W. Andreoni, S.T. Pantelides, Phys. Rev. Lett. 70, 2435 (1993)

52. S.J. Clark, G.J. Ackland, Phys. Rev. B 56, 47 (1997)

53. O.K. Al-Mushadani, R.J. Needs, Phys. Rev. B 68, 235205 (2003)

54. J. Zhu, T.D. de la Rubia, L.H. Yang, C. Mailhiot, G.H. Gilmer, Phys. Rev. B 54, 4741 (1996)

55. R. Car, P.J. Kelly, A. Oshiyama, S.T. Pantelides, Phys. Rev. Lett. 52, 1814 (1984)

56. R. Car, P. Blöchl, E. Smargiassi, Mater. Sci. Forum 8387, $433(1992)$

57. J. Zhu, L.H. Yang, C. Mailhiot, T.D. de la Rubia, G.H. Gilmer, Nucl. Instrum. Methods Phys. Res. B 102, 29 (1995)

58. W.K. Leung, R.J. Needs, G. Rajagopal, S. Itoh, S. Ihara, Phys. Rev. Lett. 83, 2351 (1999)

59. R.J. Needs, J. Phys.: Condens. Matter 11, 10437 (1999)

60. M. Tang, L. Colombo, J. Zhu, T.D. de la Rubia, Phys. Rev. B 55, 14279 (1997)

61. L.J. Munro, D.J. Wales, Phys. Rev. B 59, 3969 (1999)

62. C.Z. Wang, C.T. Chan, K.M. Ho, Phys. Rev. Lett. 66, $189(1991)$
63. E.G. Song, E. Kim, Y.H. Lee, Y.G. Hwang, Phys. Rev. B 48, 1486 (1993)

64. A. Jääskeläinen, L. Colombo, R. Nieminen, Phys. Rev. B 64, 233203 (2001)

65. D.A. Richie, J. Kim, S.A. Barr, K.R.A. Hazzard, R. Hennig, J.W. Wilkins, Phys. Rev. Lett. 92, 045501 (2004)

66. T.J. Lenosky, J.D. Kress, I. Kwon, A.F. Voter, B. Edwards, D.F. Richards, S. Yang, J.B. Adams, Phys. Rev. B 55, 1528 (1997)

67. P.J. Ungar, T. Halicioglu, W.A. Tiller, Phys. Rev. B 50, 7344 (1994)

68. K. Nishihira, T. Motooka, Phys. Rev. B 66, 233310 (2002)

69. I.P. Batra, F.F. Abraham, S. Ciraci, Phys. Rev. B 35, 9552 (1987)

70. H.R. Schober, Phys. Rev. B 39, 13013 (1989)

71. D. Maroudas, R.A. Brown, Appl. Phys. Lett. 62, 172 (1993)

72. D. Maroudas, R.A. Brown, Phys. Rev. B 47, 15562 (1993)

73. D. Maroudas, S.T. Pantelides, Chem. Eng. Sci. 49, 3001 (1994)

74. G.H. Gilmer, T.D. de la Rubia, D.M. Stock, M. Jaraiz, Nucl. Instrum. Methods Phys. Res. B 102, 247 (1995)

75. T. Sinno, K. Jiang, R.A. Brown, Appl. Phys. Lett. 68, $3028(1996)$

76. M. Nastar, V.V. Bulatov, S. Yip, Phys. Rev. B 53, 13521 (1996)

77. M. Hane, T. Ikezawa, A. Furukawa, IEICE Trans. Electron. E83-C, 1247 (2000)

78. L.A. Marqués, L. Pelaz, P. Castrillo, J. Barbolla, Phys. Rev. B 71, 085204 (2005)

79. H. Föll, U. Gösele, B.O. Kolbesen, J. Cryst. Growth $\mathbf{5 2}$ 907 (1981)

80. R. Falster, V.V. Voronkov, F. Quast, Phys. Status Solidi B 222, 219 (2000)

81. T. Sinno, E. Dornberger, W. von Ammon, R.A. Brown, F. Dupret, Mater. Sci. Eng. R 28, 149 (2000)

82. S. Lee, G.S. Hwang, Phys. Rev. B 78, 045204 (2008)

83. A. Bongiorno, L. Colombo, T.D.D. la Rubia, Europhys. Lett. 43, 695 (1998)

84. T.E.M. Staab, A. Sieck, M. Haugk, M. Puska, T. Frauenheim, H. Leipner, Phys. Rev. B 65, 115210 (2002)

85. J.L. Hastings, S.K. Estreicher, P. Fedders, Phys. Rev. B 56, 10215 (1997)

86. S. Lee, G.S. Hwang, Phys. Rev. B 78, 125310 (2008)

87. E. Domberger, D. Temmler, W. von Ammon, J. Electrochem. Soc. 149, G226 (2002)

88. R. Winkler, G. Behnke, (The Electrochemical Society, Pennington, NJ, 1994), vol. 94, p. 673

89. E.G. Roth, O.W. Holland, J.L. Duggan, in Proceedings of Application of Accelerators in Research and Industry, Pts 1 and 2, vol. 475 (1999), vol. 475, p. 804

90. J. Xu, E.G. Roth, O.W. Holland, A.P. Mills, R. Suzuki, Appl. Phys. Lett. 74, 997 (1999)

91. A.J. Smith, N.E.B. Cowern, R. Gwilliam, B.J. Sealy, B. Colombeau, E.J.H. Collart, S. Gennaro, D. Giubertoni, M. Bersani, M. Barozzi, Appl. Phys. Lett. 88, $082112(2006)$

92. E. Bruno, S. Mirabella, F. Priolo, E. Napolitani, C. Bongiorno, V. Raineri, J. Appl. Phys. 101, 023515 (2007)

93. M. Itsumi, H. Akiya, T. Ueki, M. Tomita, M. Yamawaki, J. Appl. Phys. 78, 5984 (1995)

94. D.J. Eaglesham, A. E.White, L.C. Feldman, D.C. Jacobson, Phys. Rev. Lett. 70, 1643 (1993) 
95. M. Prasad, T. Sinno, Phys. Rev. B 68, 045206 (2003)

96. J.P. Goss, P. Briddon, R. Jones, J. Phys.: Condens. Matter 16, 3311 (2004)

97. V.C. Venezia, L. Pelaz, H.J.L. Gossmann, T.H. haynes, C.S. Rafferty, Phys. Rev. Lett. 79, 1273 (2001)

98. R. Kalyanaraman, T.E. Haynes, O.W. Holland, H.J.L. Gossmann, C.S. Rafferty, G.H. Gilmer, Appl. Phys. Lett. 79, 1983 (2001)

99. L. Colombo, Physica B 273-274, 458 (1999)

100. M.P. Chichkine, M.M.D. Souza, E.M.S. Narayanan, Phys. Rev. Lett. 88, 085501 (2002)

101. I. Martín-Bragado, M. Jaraiz, P. Castrillo, R. Pinacho, J. Barbolla, M.M.D. Souza, Phys. Rev. B 68, 195204 (2003)

102. W. Ng, M. Lourenco, R. Gwilliam, S. Ledain, G. Shao, K. Homewood, Nature 410, 192 (2001)

103. N. Arai, S. Takeda, M. Kohyama, Phys. Rev. Lett. 78, 4265 (1997)

104. S. Takeda, Jap. J. Appl. Phys. 30, L639 (1991)

105. F. Cristiano, N. Cherkashin, X. Hebras, P. Calvo, Y. Lamrani, E. Scheid, B. de Mauduit, B. Colombeau, W. Lerch, S. Paul, A. Claverie, Nucl. Instrum. Methods Phys. Res. B 216, 46 (2004)

106. J. Li, K. Jones, Appl. Phys. Lett. 73, 3648 (1998)

107. H. Park, J.W. Wilkins, Phys. Rev. B 79, 241203 (2009)

108. F. Cristiano, J. Grisolia, B. Colombeau, M. Omri, B. de Mauduit, A. Claverie, F.L. Giles, J. Appl. Phys. 87, $8420(2000)$

109. K. Jones, J. Liu, L. Zhang, V. Krishnamoorthy, R. DeHoff, Nucl. Instrum. Methods Phys. Res. B 106, 227 (1995)

110. D. Eaglesham, P. Stolk, H.J. Gossmann, T. Haynes, J. Poate, Nucl. Instrum. Methods Phys. Res. B 106, 191 (1995)

111. L. Pelaz, G.H. Gilmer, M. Jaraiz, S.B. Herner, H.J. Gossmann, D.J. Eaglesham, G. Hobler, C.S. Rafferty, J. Barbolla, Appl. Phys. Lett. 73, 1421 (1998)

112. L. Pelaz, G.H. Gilmer, V.C. Venezia, H.J. Gossmann, M. Jaraiz, J. Barbolla, Appl. Phys. Lett. 74, 2017 (1999)

113. L. Pelaz, G.H. Gilmer, H.J. Gossmann, C.S. Rafferty, M. Jaraiz, J. Barbolla, Appl. Phys. Lett. 74, 3657 (1999)

114. M. Aboy, L. Pelaz, L.A. Marqués, L. Enríquez, J. Barbolla, J. Appl. Phys. 94, 1013 (2003)

115. M. Aboy, L. Pelaz, E. Bruno, S. Mirabella, S. Boninelli, J. Appl. Phys. 110, 073525 (2011)

116. G. Davies, Physics Reports 176, 83 (1989)

117. G. Davies, E. Lightowlers, Z. Ciechanowska, J. Appl. Phys. C: Solid State Physics 20, 191 (1987)

118. S. Charnvanichborikarn, B. Villis, B. Johnson, J. WongLeung, J. McCallum, J. Williams, C. Jagadish, Appl. Phys. Lett. 96, 051906 (2010)

119. M. Nakamura, S. Nagai, Phys. Rev. B 66, 155204 (2002)

120. B. Coomer, J. Goss, R. Jones, S. Oberg, P. Briddon, Physica B: Physics of Condensed Matter 273, 505 (1999)

121. A. Carvalho, R. Jones, J. Coutinho, P. Briddon, Phys. Rev. B 72, 155208 (2005)

122. G.M. Lopez, V. Fiorentini, Phys. Rev. B 69, 155206 (2004)

123. P.A. Schultz, Phys. Rev. Lett. 96, 246401 (2006)

124. T.E. Haynes, D.J. Eaglesham, P. Stolk, H. Gossmann, D. Jacobson, J. Poate, Appl. Phys. Lett. 69, 1376 (1996)

125. C.S. Rafferty, G.H. Gilmer, M. Jaraiz, D.J. Eaglesham, H.J. Gossman, Appl. Phys. Lett. 68, 2395 (1996)

126. A. Ural, P.B. Griffin, J.D. Plummer, J. Appl. Phys. 85, 6440 (1999)
127. H.J. Gossmann, T.E. Haynes, P.A. Stolk, D.C. Jacobson, G.H. Gilmer, J.M. Poate, H.S. Luftman, T.K. Mogi, M.O. Thompson, Appl. Phys. Lett. 71, 3862 (1997)

128. C.S. Nichols, C.G.V. de Walle, S.T. Pantelides, Phys. Rev. B 40, 5484 (1989)

129. B. Sadigh, T.J. Lenosky, S.K. Theiss, M.J. Caturla, Phys. Rev. Lett. 83, 4341 (1999)

130. W. Windl, M.M. Bunea, R. Stumpf, S.T. Dunham, M.P. Masquelier, Phys. Rev. Lett. 83, 4345 (1999)

131. G.D. Watkins, Phys. Rev. B 12, 5824 (1975)

132. P. Alippi, L. Colombo, P. Ruggerone, A. Sieck, G. Seifert, T. Frauenheim, Phys. Rev. B 64, 075207 (2001)

133. D. De Salvador, E. Napolitani, S. Mirabella, G. Bisognin, G. Impellizzeri, A. Carnera, F. Priolo, Phys. Rev. Lett. 97, 255902 (2006)

134. D. De Salvador, E. Napolitani, G. Bisognin, M. Pesce, A. Carnera, E. Bruno, G. Impellizzeri, S. Mirabella, F. Priolo, Phys. Rev. B 81, 045209 (2010)

135. S. Mirabella, D.D. Salvador, E. Napolitani, E. Bruno, F. Priolo, J. Appl. Phys. 113, 031101 (2013)

136. B. Pawlak, W. Vandervorst, A. Smith, C. N.E.B, C. B, X. Pages, Appl. Phys. Lett. 86, 101913 (2005)

137. V.C. Venezia, R. Duffy, L. Pelaz, M. Aboy, A. Heringa, P.B. Griffin, C.C. Wang, M.J.P. Hopstaken, Y. Tamminga, T. Dao, B. Pawlak, F. Roozeboom, in IEEE International Electron Devices Meeting (2003), pp. 20.3.1-4

138. D. De Salvador, G. Bisognin, M.D. Marino, E. Napolitani, A. Carnera, H. Graoui, M. Foad, F. Boscherini, S. Mirabella, Appl. Phys. Lett. 89, 241901 (2006)

139. A. Mattoni, L. Colombo, Phys. Rev. B 69, 45204 (2004)

140. S. Mirabella, D. De Salvador, E. Bruno, E. Napolitani, E. Pecora, S. Boninelli, F. Priolo, Phys. Rev. Lett. 100, 155901 (2008)

141. I. Martin-Bragado, N. Zographos, Solid-State Electron. 55, $25(2011)$

142. N.E.B. Cowern, K.T.F. Janssen, H.F.F. Jos, J. Appl. Phys. 68, 6191 (1990)

143. N.E.B. Cowern, A. Cacciato, J.S. Custer, F.W. Saris, W. Vandervorst, Appl. Phys. Lett. 68, 1150 (1996)

144. M. Aboy, L. Pelaz, L.A. Marqués, J. Barbolla, A. Mokhberi, Y. Takamura, P.B. Griffin, J.D. Plummer, Appl. Phys. Lett. 83, 4166 (2003)

145. W. Luo, P.B. Rasband, P. Clancy, B.W. Roberts, J. Appl. Phys. 84, 2476 (1998)

146. M.J. Caturla, M.D. Johnson, T.D.D. la Rubia, Appl. Phys. Lett. 72, 2736 (1998)

147. X.Y. Liu, W. Windl, M.P. Masquelier, Appl. Phys. Lett. 77, 2018 (2000)

148. T.J. Lenosky, B. Sadigh, S.K. Theiss, M.J. Caturla, T.D. de la Rubia, Appl. Phys. Lett. 77, 1834 (2000)

149. W. Luo, P. Clancy, J. Appl. Phys. 89, 1596 (2001)

150. P. Alippi, P. Ruggerone, L. Colombo, Phys. Rev. B 69, 125205 (2004)

151. M. Cogoni, A. Mattoni, B.P. Uberuaga, A.F. Voter, L. Colombo, Appl. Phys. Lett. 87, 191912 (2005)

152. F. Cristiano, X. Hebras, N. Cherkashin, A. Claverie, W. Lerch, S. Paul, Appl. Phys. Lett. 83, 5407 (2003)

153. S. Boninelli, S. Mirabella, E. Bruno, F. Priolo, F. Cristiano, A. Claverie, D. De Salvador, G. Bisognin, E. Napolitani, Appl. Phys. Lett. 91, 031905 (2007)

154. D. De Salvador, E. Napolitani, G. Bisognin, A. Carnera, E. Bruno, S. Mirabella, G. Impellizzeri, F. Priolo, Appl. Phys. Lett. 87, 221902 (2005) 
155. B.J. Pawlak, R. Surdeanu, B. Colombeau, A.J. Smith, N.E.B. Cowern, R. Lindsay, W. Vandervorst, B. Brijs, O. Richard, F. Cristiano, Appl. Phys. Lett. 84, 2055 (2004)

156. L. Pelaz, V. Venezia, H.J. Gossmann, G.H. Gilmer, A. Fiory, C.S. Rafferty, M. Jaraiz, J. Barbolla, Appl. Phys. Lett. 75, 662 (1999)

157. S. Mirabella, E. Bruno, F. Priolo, D. De Salvador, E. Napolitani, A.V. Drigo, A. Carnera, Appl. Phys. Lett. 83, 680 (2003)

158. K. Jones, R. Elliman, M. Petravic, P. Kringhoj, Appl. Phys. Lett. 68, 3111 (1996)

159. A. Armigliato, D. Nobili, P. Ostoja, M. Servidori, S. Solmi, in Semiconductor Silicon 1977, vol. 77-2, ed. by H. Huff, E. Sirtl (The Electrochemical Society, Princeton, NJ, 1977), vol. 77-2, p. 638

160. J. Schmidt, A.G. Aberle, R. Hezel, Proc. 26th IEEE PVSC 13 (1997)

161. S.W. Glunz, S. Rein, J.Y. Lee, W. Warta, J. Appl. Phys. 90, 2397 (2001)

162. A. Cuevas, M.J. Kerr, C. Samundsett, Appl. Phys. Lett. 81, $4952(2002)$

163. D. Macdonald, L.J. Geerligs, Appl. Phys. Lett. 85, 4061 (2004)

164. Y. Komatsu, V.D. Mihailetchi, L.J. Geerligs, B. van Dijk, J.B. Rem, M. Harris, Solar Energy Mater. Solar Cells 93, 750 (2009)

165. R. Kopecek, J. Libal, A. Herguth, K. Peter, I. Röver, K. Wambach, B. Geerligs, Proc. 21st EU PVSEC 1264 (2006)

166. B.J. Pawlak, T. Janssens, S. Singh, I. Kuzma-Filipek, J. Robbelein, N.E. Posthuma, J. Poortmans, F. Cristiano, E. Baziz, Prog. Photovolt: Res. Appl. 20, 106 (2012) 